\title{
Outflow activity in the UCHII region G045.47+0.05
}

\author{
M. E. Ortega ${ }^{1}$, S. Paron ${ }^{1,2}$, S. Cichowolski ${ }^{1}$, M. Rubio ${ }^{3}$, and G. Dubner ${ }^{1}$ \\ ${ }^{1}$ Instituto de Astronomía y Física del Espacio (IAFE), CC 67, Suc. 28, 1428 Buenos Aires, Argentina \\ e-mail: mortega@iafe.uba.ar \\ 2 FADU - Universidad de Buenos Aires, Argentina \\ ${ }^{3}$ Departamento de Astronomía, Universidad de Chile, Casilla 36-D, Santiago, Chile
}

Received 17 April 2012 / Accepted 2 August 2012

\begin{abstract}
Aims. This work aims at investigating the molecular gas in the surroundings of the ultra-compact HII region G045.47+0.05 looking for evidence of molecular outflows.

Methods. We carried out observations towards a region of $2^{\prime} \times 2^{\prime}$ centered at RA $=19^{\mathrm{h}} 14^{\mathrm{m}} 25.6^{\mathrm{s}}$, Dec $=+11^{\circ} 09^{\prime} 27.6^{\prime \prime}(\mathrm{J} 2000)$ using the Atacama Submillimeter Telescope Experiment (ASTE; Chile) in the ${ }^{12} \mathrm{CO} J=3-2,{ }^{13} \mathrm{CO} J=3-2, \mathrm{HCO}^{+} J=4-3$, and CS $J=7-6$ lines with an angular resolution of 22". We complement these observations with public infrared data.

Results. We characterize the physical parameters of the molecular clump where G045.47+0.0 is embedded. The detection of the CS $J=7-6$ line emission in the region reveals that the ultra-compact HII region G045.47+0.0 has not completely disrupted the dense gas where it was born. The $\mathrm{HCO}^{+}$abundance observed towards G045.47+0.0 suggests there is molecular outflow activity in the region. From the analysis of the ${ }^{12} \mathrm{CO} J=3-2$ transition we report bipolar molecular outflows with a total mass of about $300 M_{\odot}$. We derive a dynamical time (flow's age) of about $10^{5} \mathrm{yr}$ for the outflow gas, in agreement with the presence of an ultra-compact HII region. We identify the source 2MASS $19142564+1109283$ as the massive protostar candidate to drive the molecular outflows. Based on the analysis of its spectral energy distribution, we infer that it is an early B-type star of about $15 M_{\odot}$. The results of this work support the scenario where the formation of massive stars, at least up to early B-type stars, is similar to that of low-mass stars.
\end{abstract}

Key words. stars: formation - ISM: jets and outflows - ISM: molecules

\section{Introduction}

Despite the importance that massive stars have in the structure and dynamic of the Galaxy, the physical processes involved in their formation are less understood than those of their low-mass counterpart. Observationally, the main problems arise from their being heavily obscured by dust, being rare, and evolving very fast, all of which makes their detection very difficult. The study of massive star formation also poses major theoretical challenges because they begin burning their nuclear fuel and radiating prodigious amounts of energy while still accreting. If the formation of massive stars is similar to that of low-mass stars (i.e. via accretion from the surrounding envelope), a mass accretion rate $\dot{M}$ of at least several orders of magnitude above the values appropriate for the low-mass star formation is required (Tan \& McKee 2002). At present, two theoretical scenarios are proposed to explain the formation of these stars: a monolithic collapse of turbulent gas on the scale of massive dense cores (Tan \& McKee 2002), which is a scaled-up version of the lowmass star formation picture, and a competitive one where accretion occurs inside the gravitational potential of a cluster-forming massive dense core (Bonnell \& Bate 2006). This last model predicts that stars located near the center of the full gravitational potential accrete at much higher rates than do isolated stars.

The detection of molecular outflows associated with both high- and low-mass young stellar objects, supports a scaled-up formation picture (Beuther 2002). In this context, the study of massive molecular outflows and the associated driving source might contribute to discern which scenario prevails in a given star-forming region.
It is well known that the generation of molecular outflows during the formation of a high-mass star is a phenomenon that can take place even when the ultra-compact HII (UCHII) region stage has been reached (Hunter et al. 1997; Qin et al. 2008). In this work we report the study of the UCHII region G045.47+0.05 (hereafter G45.47), through analysis of its associated molecular gas and searching for evidence of molecular outflows and its associated driving source. G45.47 was first detected by Wood \& Churchwell (1989) in radio continuum at $6 \mathrm{~cm}$. This object is adjacent to the extensively studied UCHII region G45.45+0.06 (hereafter G45.45), which is part of a complex of five compact HII regions (Matthews et al. 1977; Giveon et al. 2005a; Giveon et al. 2005b). Such complex and G45.47 are embedded in the molecular cloud GRSMC G045.49+00.04 (at $V_{\mathrm{LSR}} \sim 58 \mathrm{~km} \mathrm{~s}^{-1}$; Rathborne et al. 2009) and are located on the northern border of the more extended HII region named G45L in Paron et al. (2009). Based on HI absorption profiles, Kuchar \& Bania (1994) derived a kinematics distance of $8.3 \mathrm{kpc}$ for the UCHII region G45.45. As G45.47 is part of the same complex, we adopt for this object the same distance.

Caswell et al. (1995) detected a class II $\mathrm{CH}_{3} \mathrm{OH}$ maser emission at $6.6 \mathrm{GHz}$ at $V_{\mathrm{LSR}} \sim 56 \mathrm{~km} \mathrm{~s}^{-1}$ towards G45.47. Given that the $6.6 \mathrm{GHz}$ methanol maser is radiatively pumped by IR emission from the warm dust associated exclusively with massive young stellar object (MYSOs) ${ }^{1}$, their detection is useful for studying the kinematic of the gas and, in particular, to establish the systemic velocity (Cyganowski et al. 2009).

1 We define MYSOs to be young stellar objects (YSOs) that will eventually become main-sequence $\mathrm{O}$ or early B type stars $\left(M_{*} \geq 8 M_{\odot}\right)$. 


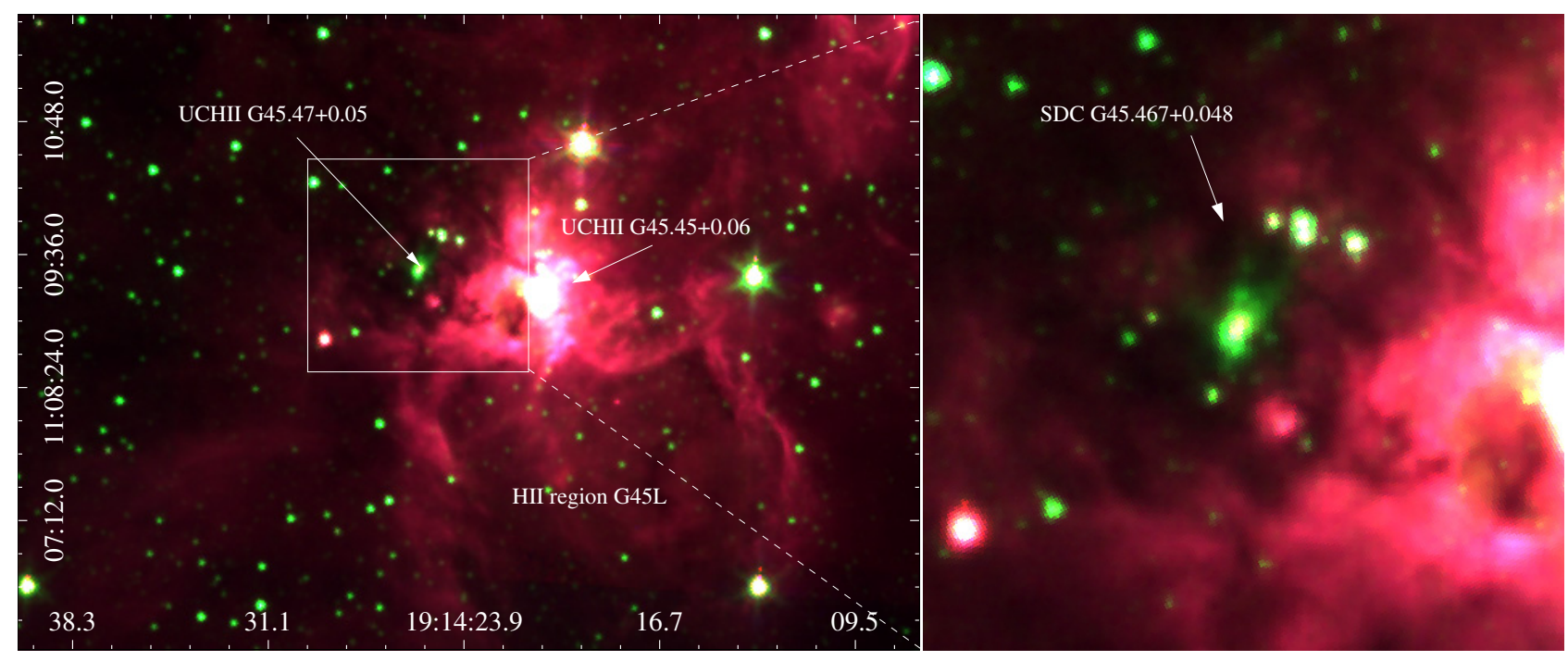

Fig. 1. Spitzer-IRAC three-color images $(3.6 \mu \mathrm{m}=$ blue, $4.5 \mu \mathrm{m}=$ green, and $8 \mu \mathrm{m}=$ red). The white box in the left panel indicates the region mapped with ASTE. A close up view of this region is shown in the right panel. Red, green, and blue scales go from 30 to 500 , from 2 to 110 , and from 1 to $80 \mathrm{MJy} / \mathrm{sr}$, respectively.

Based on high-resolution molecular line observations towards G45.47, Wilner et al. (1996) identified five $\mathrm{HCO}^{+}(1-0)$ clumps and suggest that G45.47 is in the early stages of forming an OB cluster. From an ammonia absorption study, Hofner et al. (1999) suggested that a remnant molecular core is falling into the UCHII region. Later, Cyganowski et al. (2008) identified an "extended green object" (EGO) at the position of G45.47, the EGO G45.47+0.05. Their identification as EGOs comes from the common coding of the $4.5 \mu \mathrm{m}$ band in the three-color composite Infrared Array Camera (IRAC; Fazio et al. 2004) images from the Spitzer Telescope. Extended $4.5 \mu \mathrm{m}$ emission is thought to prove there is shocked molecular gas in protostellar outflows. The association of EGOs with IRDCs and $6.7 \mathrm{GHz} \mathrm{CH}_{3} \mathrm{OH}$ maser suggests that EGOs trace the formation of massive protostars. According to Cyganowski et al. (2008), an EGOs is a MYSO with ongoing outflow activity and actively accreting.

In summary, G45.47 is a rich and complex region for studying the formation of a new generation of massive stars. In this paper we present a new study of the dense ambient medium where the UCHII region is evolving. We investigate the molecular gas through several molecular lines observed with the Atacama Submillimeter Telescope Experiment (ASTE; Chile) and characterize the central source based on public infrared data.

\section{Data}

The molecular line observations were carried out on June 12 and 13, 2011 with the $10 \mathrm{~m}$ Atacama Submillimeter Telescope Experiment (ASTE; Ezawa et al. 2004). We used the CATS345 GHz band receiver, which is a two-single band SIS receiver remotely tunable in the LO frequency range of 324-372 GHz. We simultaneously observed ${ }^{12} \mathrm{CO} \quad J=3-2$ at $345.796 \mathrm{GHz}$ and $\mathrm{HCO}^{+} J=4-3$ at $356.734 \mathrm{GHz}$, mapping a region of $2^{\prime} \times 2^{\prime}$ centered at $\mathrm{RA}=19^{\mathrm{h}} 14^{\mathrm{m}} 25.6^{\mathrm{s}}$, Dec $=+11^{\circ} 09^{\prime} 27 \cdot 6^{\prime \prime}(\mathrm{J} 2000)$. We also observed ${ }^{13} \mathrm{CO} J=3-2$ at $330.588 \mathrm{GHz}$ and $\mathrm{CS} J=7-6$ at $342.883 \mathrm{GHz}$ towards the same region. The mapping grid spacing was $20^{\prime \prime}$ in both cases, and the integration time was $20 \mathrm{~s}\left({ }^{12} \mathrm{CO}\right.$ and $\left.\mathrm{HCO}^{+}\right)$and $40 \mathrm{~s}\left({ }^{13} \mathrm{CO}\right.$ and CS) per pointing. All the observations were performed in position-switching mode. We verified that the off position
$\left(\mathrm{RA}=19^{\mathrm{h}} 14^{\mathrm{m}} 21.6^{\mathrm{s}}\right.$, Dec $\left.=+10^{\circ} 59^{\prime} 4^{\prime \prime}, \mathrm{J} 2000\right)$ was free of emission. We used the XF digital spectrometer with a bandwidth and spectral resolution set to $128 \mathrm{MHz}$ and $125 \mathrm{kHz}$. The velocity resolution was $0.11 \mathrm{~km} \mathrm{~s}^{-1}$ and the half-power beamwidth (HPBW) was 22", for all observed molecular lines. The system temperature varied from $T_{\text {sys }}=150$ to $200 \mathrm{~K}$. The main beam efficiency was $\eta_{\mathrm{mb}} \sim 0.65$. All the spectra were Hanning smoothed to improve the signal-to-noise ratio. The baseline fitting was carried out using second order polynomials for the ${ }^{12} \mathrm{CO}$ and ${ }^{13} \mathrm{CO}$ transitions and fourth order polynomials for the $\mathrm{HCO}^{+}$and $\mathrm{CS}$ transitions. The polynomia was the same for all spectra of the map of a given transition. The resulting rms noise of the observations was about $0.2 \mathrm{~K}$. The data were reduced with NEWSTAR ${ }^{2}$ and the spectra processed using the XSpec software package ${ }^{3}$.

The observations are complemented with near- and mid-IR data extracted from public databases and catalogs, which are described in the corresponding sections.

\section{Results and discussion}

Figure 1-(left) shows a composite Spitzer-IRAC three-color image $(3.6 \mu \mathrm{m}=$ blue, $4.5 \mu \mathrm{m}=$ green, and $8 \mu \mathrm{m}=$ red $)$. The extended HII region G45L is centered at RA $=19^{\mathrm{h}} 14^{\mathrm{m}} 17^{\mathrm{s}}$, Dec $=+11^{\circ} 07^{\prime} 48^{\prime \prime}(\mathrm{J} 2000)$ and is delimited by two arclike structures observed at $8 \mu \mathrm{m}$. The UCHII region G45.47 (a.k.a. EGO $\mathrm{G} 045.47+0.05)$ is the green source located at $\mathrm{RA}=19^{\mathrm{h}} 14^{\mathrm{m}} 25.6^{\mathrm{s}}$, Dec $=+11^{\circ} 09^{\prime} 27.6^{\prime \prime}(\mathrm{J} 2000)$. The white box indicates the region mapped with ASTE. A zoom of this region (Fig. 1-(right)) shows that the UCHII region is embedded in the Spitzer dark cloud SDC G45.467+0.048 (Peretto \& Fuller 2009). Infrared dark clouds (IRDCs) are dense molecular clouds that appear as extinction features against the bright mid-infrared Galactic background and have been suggested as the cold precursors to highmass stars (Rathborne et al. 2006).

\footnotetext{
2 Reduction software based on AIPS developed at NRAO, extended to treat single-dish data with a graphical user interface (GUI).

3 XSpec is a spectral line reduction package for astronomy, which was developed by Per Bergman at Onsala Space Observatory.
} 
(a)

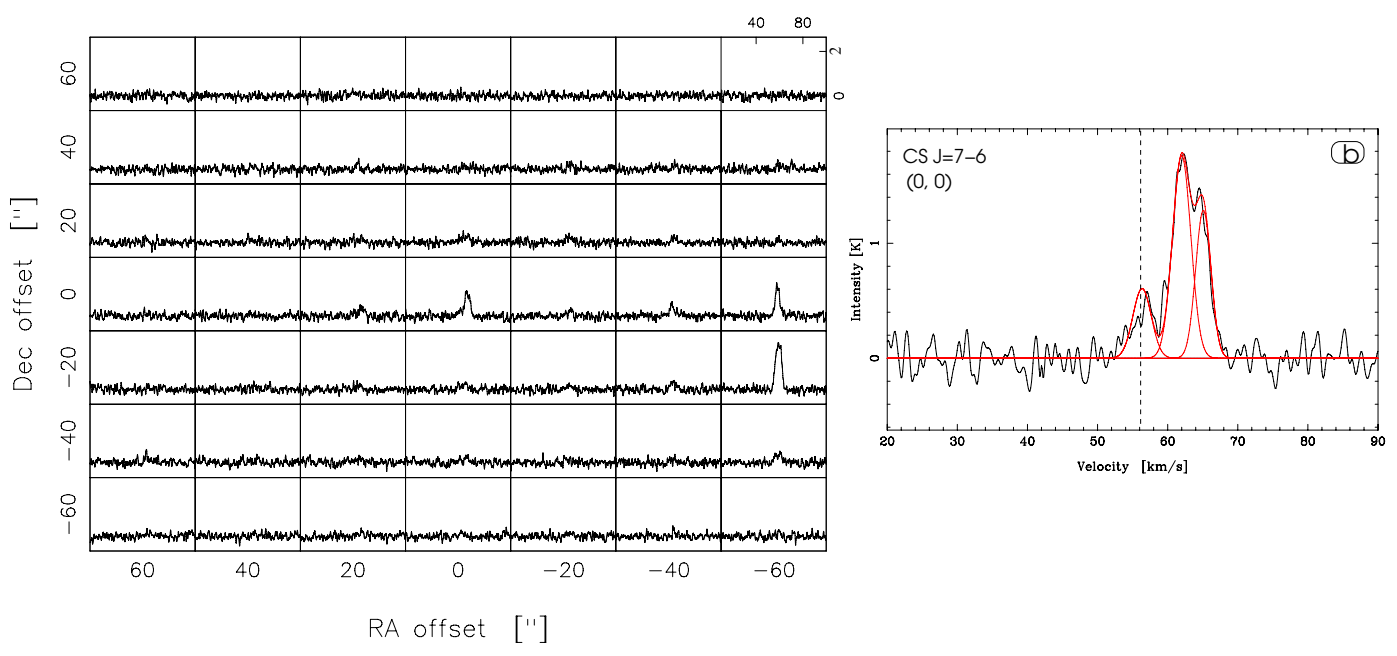

Fig. 2. a) CS $J=7-6$ spectra obtained towards the $2^{\prime} \times 2^{\prime}$ region (white box in Fig. 1-left) mapped with ASTE. b) Hanning smoothed profile of the CS $J=7-6$ line towards G45.47 at the position $(0,0)$. The dashed line indicates the systemic velocity of the gas. The spectrum was deconstructed using three Gaussians, which are shown in red.

Table 1. Emission peaks parameters derived from a Gaussian fitting for the four molecular transitions spectra on the position $(0,0)$.

\begin{tabular}{lccc}
\hline \hline Transition & $V_{\mathrm{LSR}}\left[\mathrm{km} \mathrm{s}^{-1}\right]$ & $T_{\mathrm{mb}}[\mathrm{K}]$ & $\Delta v\left[\mathrm{~km} \mathrm{~s}^{-1}\right]$ \\
\hline $\mathrm{CS} J=7-6$ & $56.3 \pm 0.9$ & $0.6 \pm 0.2$ & $3.1 \pm 0.6$ \\
& $62.0 \pm 0.8$ & $1.8 \pm 0.1$ & $3.1 \pm 0.7$ \\
& $64.8 \pm 1.1$ & $1.3 \pm 0.1$ & $2.5 \pm 0.9$ \\
\hline $\mathrm{HCO}^{+} J=4-3$ & $55.8 \pm 1.2$ & $0.4 \pm 0.2$ & $5.9 \pm 0.5$ \\
& $60.8 \pm 1.4$ & $4.4 \pm 0.8$ & $3.8 \pm 0.6$ \\
& $64.7 \pm 1.7$ & $5.1 \pm 0.6$ & $3.3 \pm 0.7$ \\
\hline${ }^{13} \mathrm{CO} J=3-2$ & $55.9 \pm 0.6$ & $5.2 \pm 0.4$ & $2.9 \pm 0.4$ \\
& $60.5 \pm 0.5$ & $11.1 \pm 0.8$ & $3.3 \pm 0.6$ \\
& $63.3 \pm 0.4$ & $6.4 \pm 0.7$ & $2.6 \pm 0.7$ \\
\hline${ }^{12} \mathrm{CO} J=3-2$ & $50.7 \pm 0.3$ & $3.0 \pm 0.2$ & $9.0 \pm 0.3$ \\
& $55.2 \pm 0.1$ & $13.1 \pm 1.1$ & $3.3 \pm 0.4$ \\
& $59.8 \pm 0.2$ & $10.6 \pm 0.8$ & $3.6 \pm 0.6$ \\
& $64.4 \pm 0.6$ & $10.6 \pm 0.7$ & $3.3 \pm 0.5$ \\
& $68.1 \pm 0.4$ & $1.3 \pm 0.2$ & $4.4 \pm 0.4$ \\
\hline
\end{tabular}

\subsection{The molecular gas}

In this section, we present the molecular results starting the description with the transitions that trace the most inner part of the clump related to $\mathrm{G} 45.47$, and moving to those that map the external layers, which may give information about the dynamic effects occurring in the gas.

Figure 2a shows the CS $J=7-6$ spectra obtained towards the $2^{\prime} \times 2^{\prime}$ region (white box in Fig. 1-left). The mapped area includes G45.47 and part of the nearby UCHII region G45.45, located at the $(0,0)$ and $(-60,-20)$ offset, respectively. As expected, the most prominent emission arises from these two positions, since the detection of the CS $J=7-6$ transition reveals the presence of warm and dense gas. Figure $2 b$ shows an enlargement of the spectrum observed at $(0,0)$. The emission related to G45.47 shows a triple-peak structure with velocity components centered at about 56 (the systemic velocity of the gas), 62, and $65 \mathrm{~km} \mathrm{~s}^{-1}$ (see Table 1) with a pronounced dip at about 59 and a less conspicuous one at $\sim 64 \mathrm{~km} \mathrm{~s}^{-1}$. The velocity component centered at $56 \mathrm{~km} \mathrm{~s}^{-1}$ is weak, with a peak about $3 \sigma$ of the rms noise level. Since the probability of superposition along the line of sight of more than one component in the CS $J=7-6$ line is very low, we suggest that the dips reveal self-absorption effects in the gas, consistent with an optically thick transition. Self-absorption features demonstrate the existence of a density gradient in the clump (Hiramatsu et al. 2007), which is a common feature of optically thick lines in the direction of embedded young stars. The depression discloses relatively cold, foreground gas that absorbs photons from warmer material behind it. The CS $J=7-6$ profile is asymmetric with respect to the dip near $v=59 \mathrm{~km} \mathrm{~s}^{-1}$ as the redshifted component is clearly brighter than the blueshifted one, suggesting that the molecular gas is expanding. This is because in an expanding cloud a line emission is composed of red- and blueshifted photons, the redshifted photons will encounter fewer absorbing material (which is expanding outward) than would blueshifted photons, hence have a greater probability of escaping (e.g. Leung 1978; Zhou 1992; Lehtinen 1997).

Figure 3 shows the velocity channel maps of the CS $J=$ 7-6 emission averaged every $1 \mathrm{~km} \mathrm{~s}^{-1}$. The presence of molecular gas associated with G45.47 can be noticed in the velocity range going from $\sim 55$ to $\sim 65 \mathrm{~km} \mathrm{~s}^{-1}$. The other most conspicuous molecular condensation, partially observed, corresponds to the gas related to G45.45 and has a central velocity of $\sim 59 \mathrm{~km} \mathrm{~s}^{-1}$.

Figure 4-a shows the $\mathrm{HCO}^{+} J=4-3$ spectra obtained towards the same region. As in the case of the CS $J=7-6$ spectrum, it can be seen that the $\mathrm{HCO}^{+}$emission towards $(0,0)$ can be deconstructed in three velocity components, a very weak one centered at about $56 \mathrm{~km} \mathrm{~s}^{-1}$ (intensity of about $2 \sigma$ ), plus two brighter ones at 61 and $65 \mathrm{~km} \mathrm{~s}^{-1}$, with only one dip centered at $\sim 63 \mathrm{~km} \mathrm{~s}^{-1}$.

From Fig. 4-a it can be noticed that the $\mathrm{HCO}^{+}$spectrum on position $(-60,-20)$, which is at the position of the UCHII region $\mathrm{G} 45.45$, has also a dip at about $60 \mathrm{~km} \mathrm{~s}^{-1}$ similar to what is observed in the CS $J=7-6$ line towards the same position.

The $\mathrm{HCO}^{+}$spectra also show evidence of emission near position $(60,-40)$. This molecular feature coincides in position with an infrared (IR) source located at RA $=19^{\mathrm{h}} 14^{\mathrm{m}} 27.7^{\mathrm{s}}$, Dec $=+11^{\circ} 08^{\prime} 33^{\prime \prime}(J 2000)$ (see Fig. 1). Since this $\mathrm{HCO}^{+}$spectrum has the same kinematic structure as the spectrum towards $(-60,-20)$ including a dip at the same velocity of about 

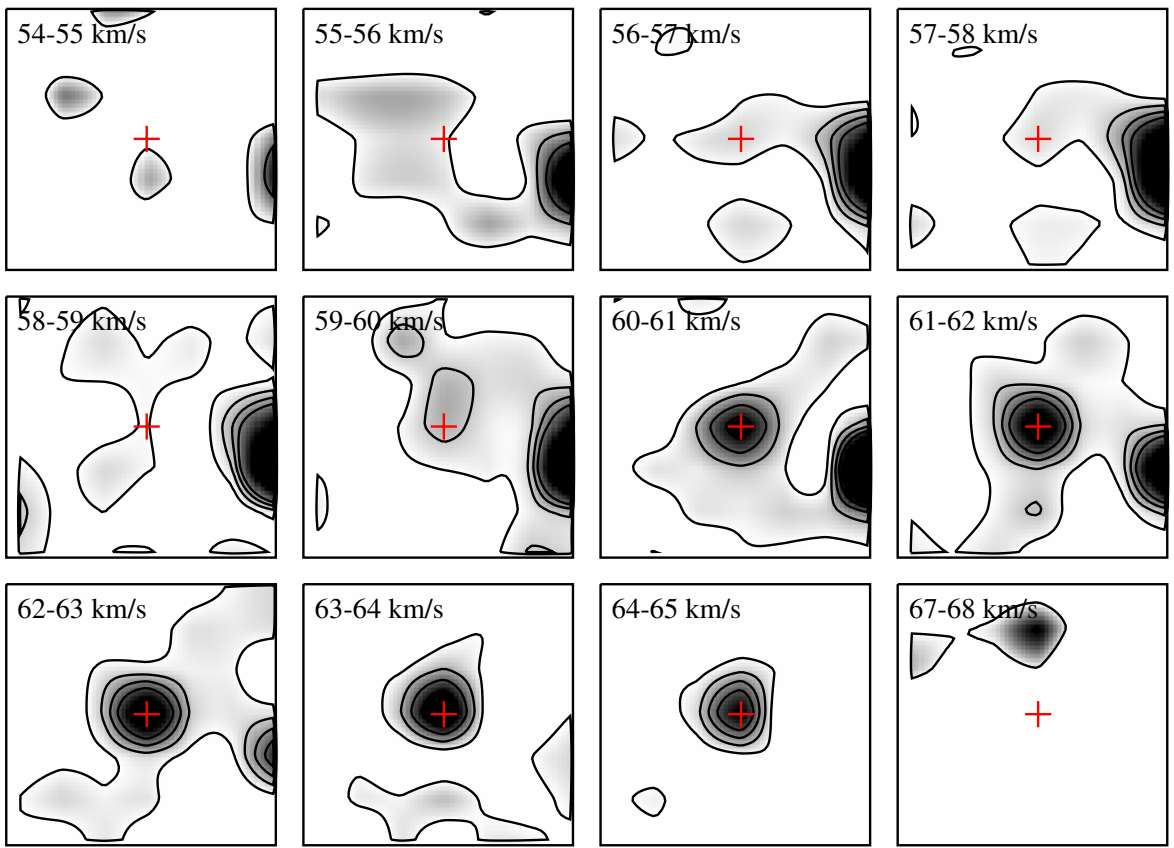

Fig. 3. Velocity channel maps of the CS $J=7-6$ emission averaged every $1 \mathrm{~km} \mathrm{~s}^{-1}$. Grayscale goes from 0.15 (about $3 \sigma$ rms noise of an averaged channel map) up to $1.8 \mathrm{~K}$. Contours levels are at $0.15,0.3,0.45$, and $0.6 \mathrm{~K}$. The red cross represents the position of G45.47.

(a)

$\mathrm{HCO}+\mathrm{J}=4-3$

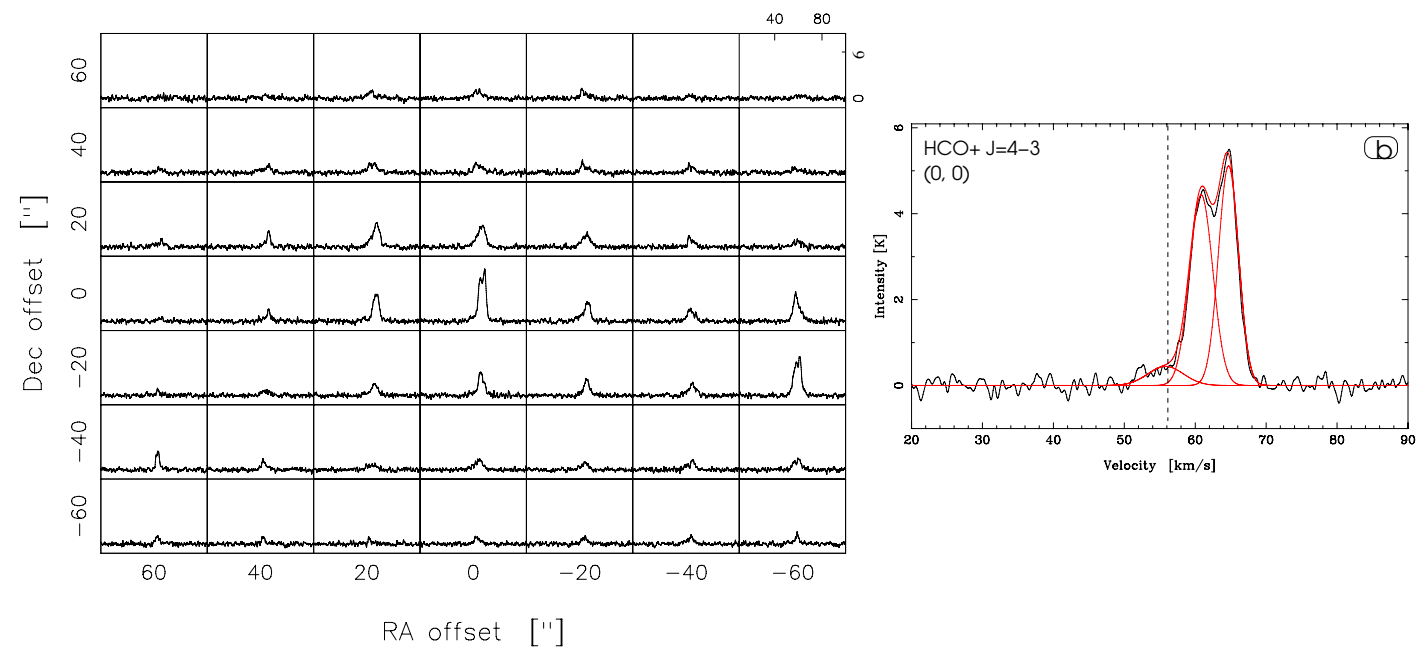

Fig. 4. a) $\mathrm{HCO}^{+} J=4-3$ spectra obtained towards the $2^{\prime} \times 2^{\prime}$ region (white box in Fig. 1-left) mapped with ASTE. b) Hanning smoothed profile of the $\mathrm{HCO}^{+} J=4-3$ line towards the position $(0,0)$ where it is G45.47. The dashed line indicates the systemic velocity of the gas. The spectrum was deconstructed using three Gaussians, which are shown in red.

$60 \mathrm{~km} \mathrm{~s}^{-1}$, we can conclude that this IR source is embedded in a molecular filament that must be connected with G45.45.

Figure 5 shows the velocity channel maps of the $\mathrm{HCO}^{+} J=$ 4-3 emission averaged every $1 \mathrm{~km} \mathrm{~s}^{-1}$. The $\mathrm{HCO}^{+} J=$ 4-3 emission related to G45.47 is visible between $\sim 55$ and $\sim 68 \mathrm{~km} \mathrm{~s}^{-1}$, while the emission associated with G45.45 goes from $\sim 54$ to $\sim 63 \mathrm{~km} \mathrm{~s}^{-1}$, respectively. Between $\sim 56$ and $\sim 60 \mathrm{~km} \mathrm{~s}^{-1}$ part of the molecular condensation related to the IR source mentioned above can be appreciated.

Figure 6 shows the ${ }^{13} \mathrm{CO} J=3-2$ spectra towards the same region. The ${ }^{13} \mathrm{CO}$ emission towards $(0,0)$ can be decomposed into three velocity components centered at about 56 (the systemic velocity of the gas), 60 , and $63 \mathrm{~km} \mathrm{~s}^{-1}$, while only one dip centered at $\sim 58 \mathrm{~km} \mathrm{~s}^{-1}$ can be appreciated. The depression at $58 \mathrm{~km} \mathrm{~s}^{-1}$ can be observed in almost all the ${ }^{13} \mathrm{CO} J=3-2$ spectra in the region. Figure 7 shows the velocity channel maps of the ${ }^{13} \mathrm{CO} J=3-2$ emission averaged every $1 \mathrm{~km} \mathrm{~s}^{-1}$.

The ${ }^{12} \mathrm{CO} J=3-2$ spectra (Fig. 8) exhibit more complicated behavior. The profile towards the position $(0,0)$ has a triplepeak structure with components centered at about 55, 60, and $64 \mathrm{~km} \mathrm{~s}^{-1}$ and two dips at about 58 and $62 \mathrm{~km} \mathrm{~s}^{-1}$. This spectrum is broadened, suggesting outflow activity in the region with the blue wing centered near the position $(20,20)$ and the red wing around the $(0,-40)$ offset.

In Fig. 9 we show the velocity channel maps of the ${ }^{12} \mathrm{CO} J=$ 3-2 emission averaged every $2.1 \mathrm{~km} \mathrm{~s}^{-1}$. Among all the observed molecular condensations, we draw attention to the clumps related to G45.47 and G45.45. The molecular clump related to G45.47 is observed between $\sim 62$ and $66 \mathrm{~km} \mathrm{~s}^{-1}$ and is seen slightly shifted to the northwest between $\sim 58$ and $62 \mathrm{~km} \mathrm{~s}^{-1}$. The 

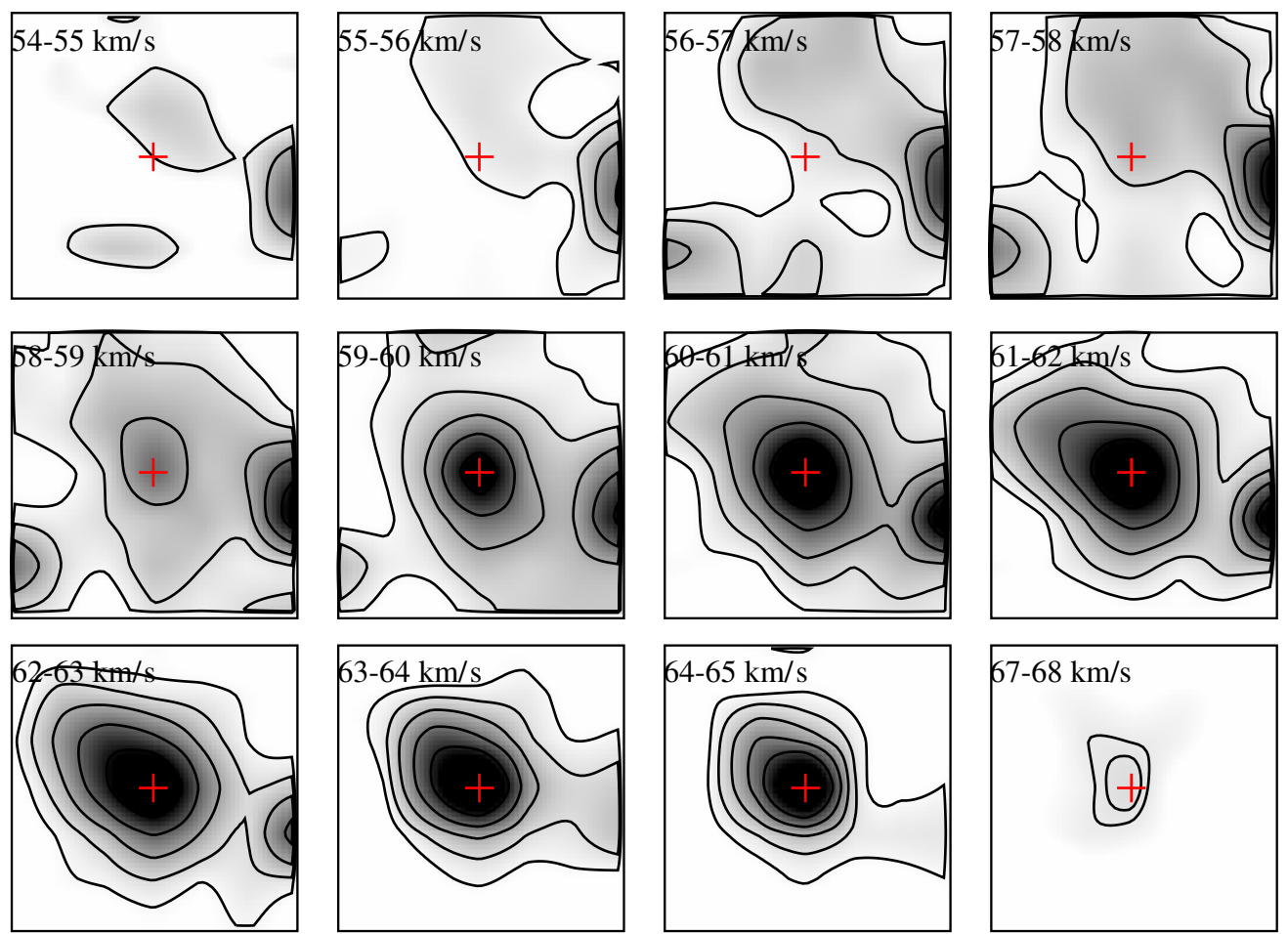

Fig. 5. Velocity channel maps of the $\mathrm{HCO}^{+} J=4-3$ emission averaged every $1 \mathrm{~km} \mathrm{~s}^{-1}$. Grayscale goes from 0.15 (about $3 \sigma$ rms noise of an averaged channel map) to $2.5 \mathrm{~K}$. Contours levels are at $0.15,0.5,0.8,1.3$, and $1.9 \mathrm{~K}$. The red cross represents the position of G45.47.

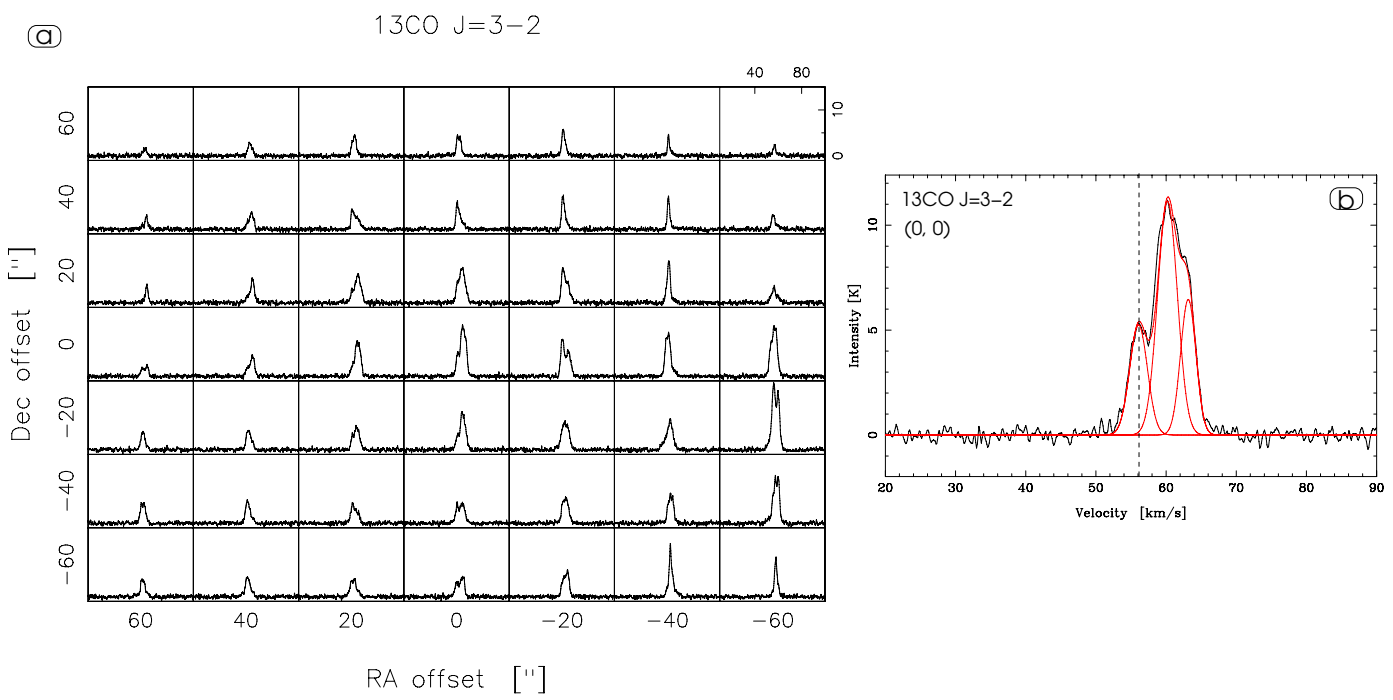

Fig. 6. a) ${ }^{13} \mathrm{CO} J=3-2$ spectra obtained towards the $2^{\prime} \times 2^{\prime}$ region (white box in Fig. 1-left) mapped with ASTE. b) Hanning smoothed profile of the ${ }^{13} \mathrm{CO} J=3-2$ line towards G45.47. The dashed line indicates the systemic velocity of the gas. The spectrum was deconstructed using three Gaussians, which are shown in red.

clump associated with G45.45 (partially observed) is centered at $\mathrm{RA}=19^{\mathrm{h}} 14^{\mathrm{m}} 22^{\mathrm{s}}$, Dec $=+11^{\circ} 09^{\prime} 20^{\prime \prime}(\mathrm{J} 2000)$ in the velocity interval going from 47 to $63 \mathrm{~km} \mathrm{~s}^{-1}$. Although both molecular condensations have different associated velocity ranges, they are connected through the extended emission shown in the velocity interval going from 50 to $65 \mathrm{~km} \mathrm{~s}^{-1}$. The molecular gas related to the spectral wings appears as two conspicuous molecular features centered at RA $=19^{\mathrm{h}} 14^{\mathrm{m}} 27^{\mathrm{s}}$, Dec $=+11^{\circ} 09^{\prime} 45^{\prime \prime}(\mathrm{J} 2000)$ between 35 and $53 \mathrm{~km} \mathrm{~s}^{-1}$ and at $\mathrm{RA}=19^{\mathrm{h}} 14^{\mathrm{m}} 26^{\mathrm{s}}$, Dec $=+11^{\circ} 08^{\prime} 45^{\prime \prime}(\mathrm{J} 2000)$ between 65 and $76 \mathrm{~km} \mathrm{~s}^{-1}$. These features are discussed further in Sect. 3.3.
The four transitions have, within errors, the same main velocity components at about 55,60 , and $64 \mathrm{~km} \mathrm{~s}^{-1}$ towards the position $(0,0)$. By the other hand, the velocity components observed at about 51 and $68 \mathrm{~km} \mathrm{~s}^{-1}$ in the ${ }^{12} \mathrm{CO} J=3-2$ spectrum are not detected in the three other lines. Table 1 lists the emission peaks parameters derived from a Gaussian fitting for the four molecular transitions on the position $(0,0)$, and $T_{\mathrm{mb}}$ represents the peak brightness temperature and $V_{\text {LSR }}$ the central velocity referred to the Local Standard of Rest. Errors are formal $1 \sigma$ value for the model of Gaussian line shape. All the spectra towards the $(0,0)$ position have the same self-absorption dip at 

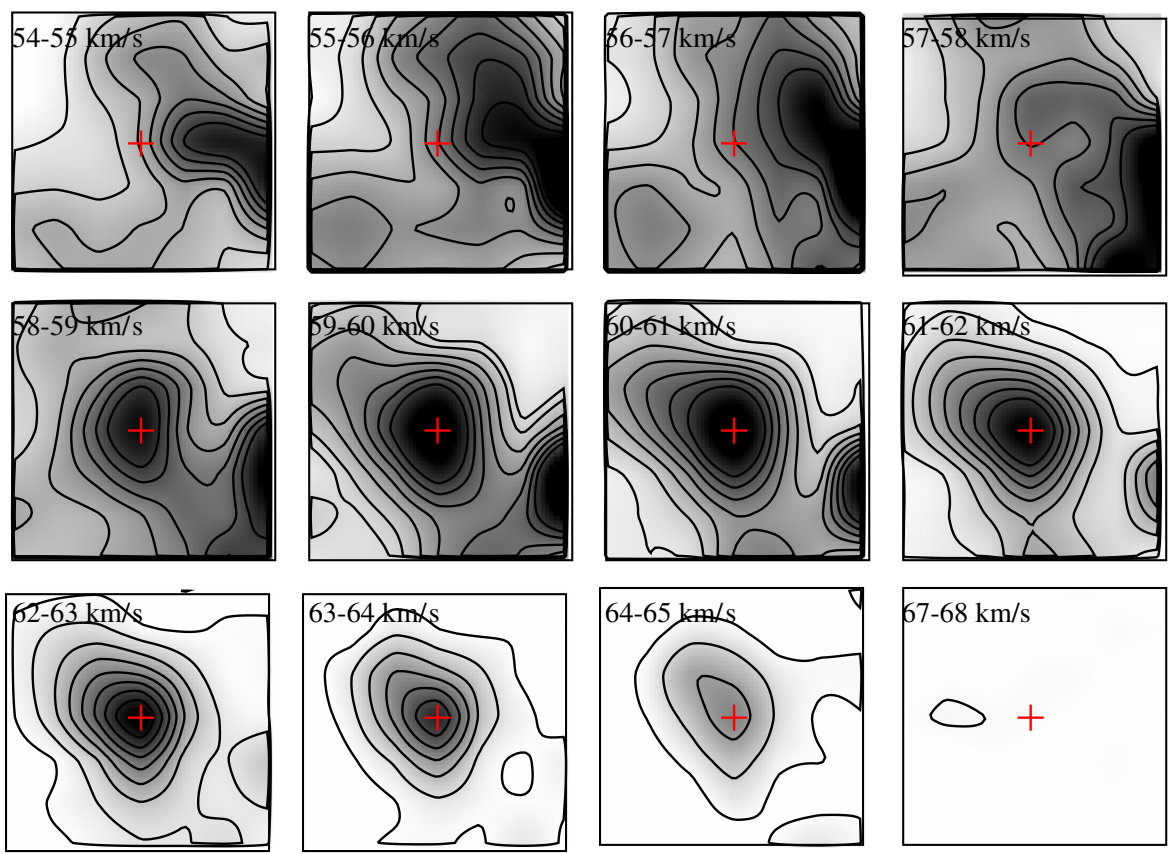

Fig. 7. Velocity channel maps of the ${ }^{13} \mathrm{CO} J=3-2$ emission averaged every $1 \mathrm{~km} \mathrm{~s}^{-1}$. Grayscale goes from 0.15 up to $9 \mathrm{~K}$. Contours levels are at $0.6,2,4,5,6,7,8$, and $9 \mathrm{~K}$. The red cross represents the position of G45.47.

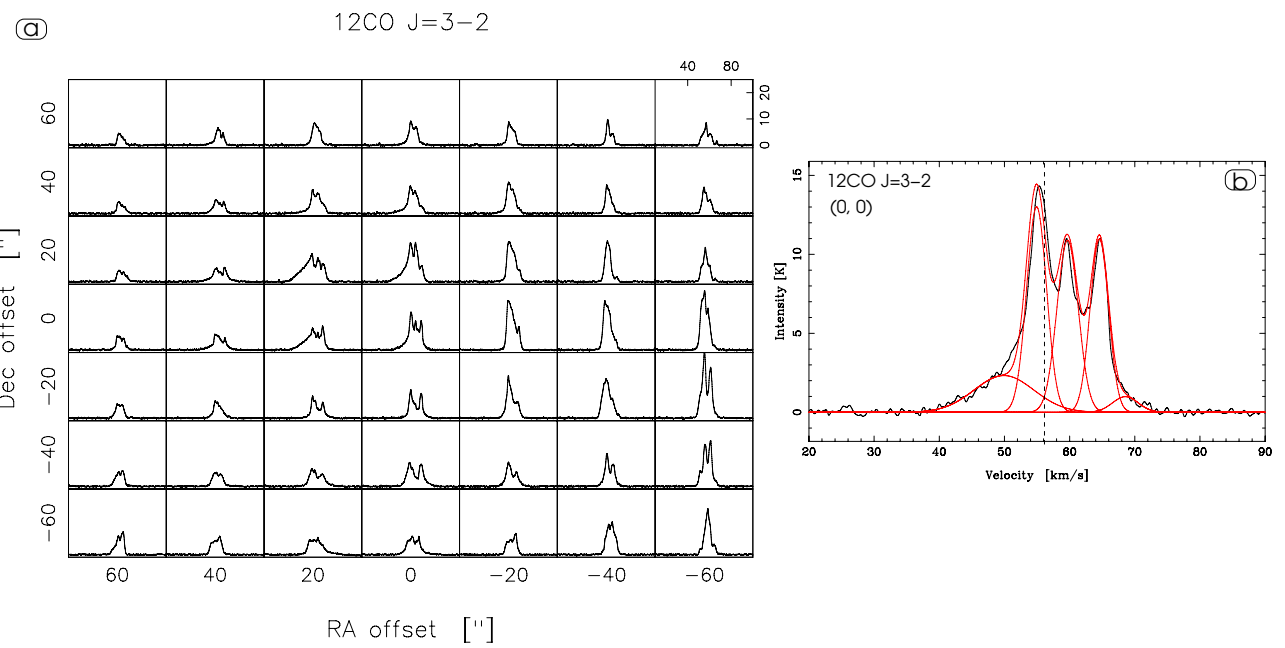

Fig. 8. a) ${ }^{12} \mathrm{CO} J=3-2$ spectra obtained towards the $2^{\prime} \times 2^{\prime}$ region (white box in Fig. 1-left) mapped with ASTE. b) Hanning smoothed profile of the ${ }^{12} \mathrm{CO} J=3-2$ line towards G45.47. The dashed line indicates the systemic velocity of the gas. The spectrum was deconstructed using five Gaussians, which are shown in red.

about $58-59 \mathrm{~km} \mathrm{~s}^{-1}$, which corresponds to the central velocity of the molecular cloud GRSMC $045.49+00.04$ where G45.47 is embedded. Even more, this spectral feature is observed in all transitions towards the region $\mathrm{G} 45.45$ and the IR source located at RA $=19^{\mathrm{h}} 14^{\mathrm{m}} 27.7^{\mathrm{s}}$, Dec $=+11^{\circ} 08^{\prime} 33^{\prime \prime}(\mathrm{J} 2000)$ (see Fig. 1).

\subsection{Column density and mass estimates of the molecular clump associated with G45.47}

We estimate the ${ }^{13} \mathrm{CO} J=3-2$ opacity, $\tau_{13}$, based on the following equation:

$\tau_{13}=-\ln \left(1-\frac{T_{\text {peak }}\left({ }^{13} \mathrm{CO}\right)}{T_{\text {peak }}\left({ }^{12} \mathrm{CO}\right)}\right)$,

where we consider $T_{\text {peak }}$ from the position $(0,0)$. We obtain, $\tau_{13} \sim 1.9$, which reveals that the ${ }^{13} \mathrm{CO} J=3-2$ emission is optically thick towards G45.47, in agreement with the observed profile towards $(0,0)$ offset (see Fig. 6$)$.

The excitation temperature, $T_{\mathrm{ex}}$, of the ${ }^{13} \mathrm{CO} J=3-2$ line was estimated from

$$
{ }^{13} T_{\text {peak }}=\frac{h v}{k}\left(\frac{1}{\mathrm{e}^{h v / k T_{\mathrm{ex}}}-1}-\frac{1}{\mathrm{e}^{h v / k T_{\mathrm{BG}}}-1}\right) \times\left(1-\mathrm{e}^{-\tau_{13}}\right)
$$

where $h v / k=15.87$ for this line. Assuming $T_{\mathrm{BG}}=2.7 \mathrm{~K}$, and considering the peak brightness temperature for the ${ }^{13} \mathrm{CO} J=$ $3-2$ at $(0,0)$ offset, ${ }^{13} T_{\text {peak }}=11.15 \mathrm{~K}$, we derive a $T_{\text {ex }} \sim 20 \mathrm{~K}$ for the ${ }^{13} \mathrm{CO} J=3-2$ line.

Finally, using the RADEX ${ }^{4}$ code (van der Tak et al. 2007) we derive the ${ }^{13} \mathrm{CO} J=3-2$ column density and the $\mathrm{H}_{2}$ volume density. The RADEX model uses the mean escape probability approximation for the radiative transfer equation.

4 http://www.sron.rug.nl/ vdtak/radex/radex.php 


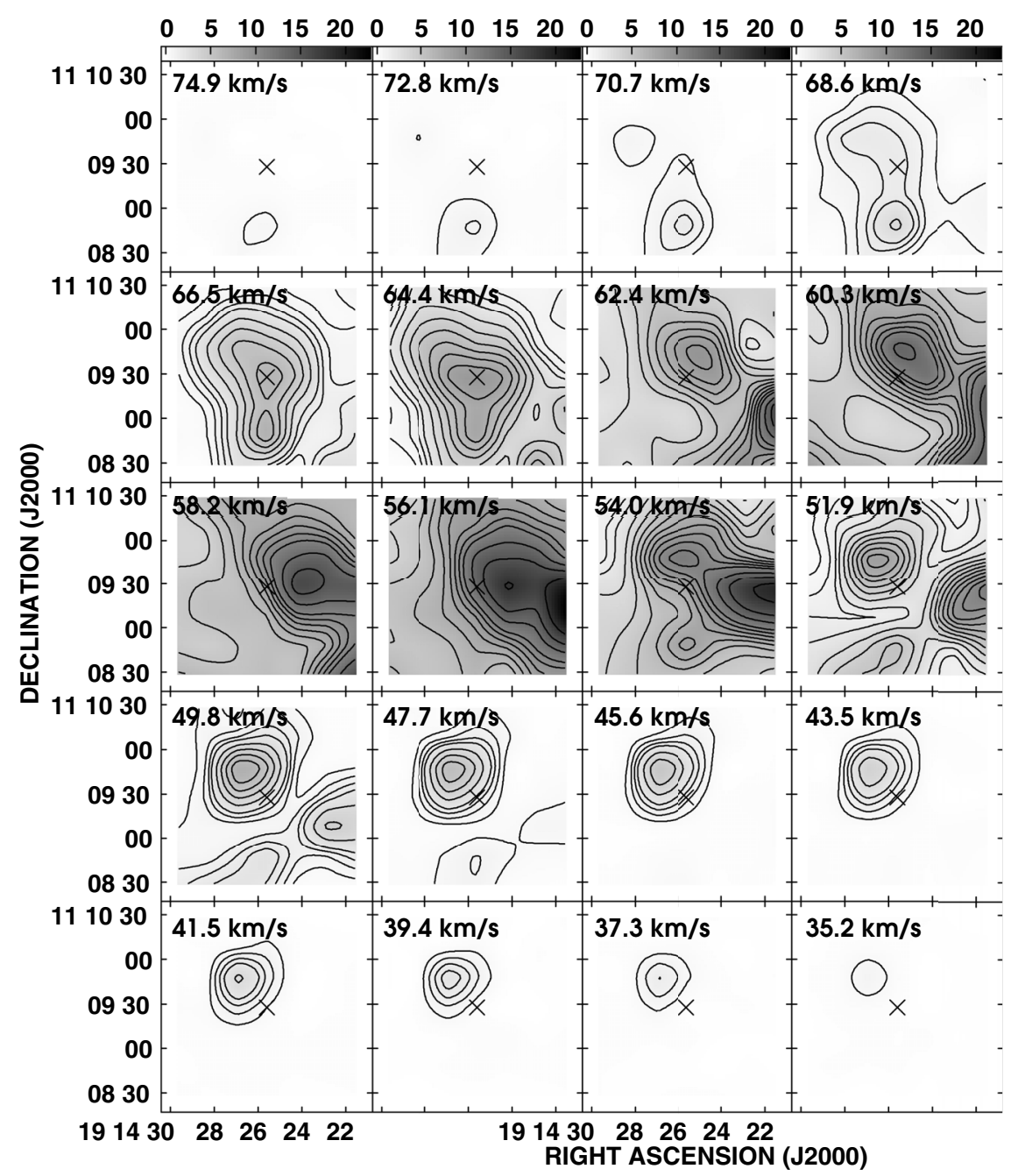

Fig. 9. Velocity channel maps of the ${ }^{12} \mathrm{CO} J=3-2$ emission averaged every $2.1 \mathrm{~km} \mathrm{~s}^{-1}$. The given velocities correspond to the higher velocity of each interval. Grayscale goes up to $23 \mathrm{~K}$. Contours are above the $5 \sigma$ of the rms noise level. The cross indicates the position of G45.47.

Adopting $T_{\mathrm{ex}} \sim 20 \mathrm{~K}, \tau_{13} \sim 1.9$, and ${ }^{13} T_{\text {peak }}=11.15 \mathrm{~K}$, we obtain a ${ }^{13} \mathrm{CO}$ column density $N\left({ }^{13} \mathrm{CO}\right) \sim 2.8 \times 10^{17} \mathrm{~cm}^{-2}$ and $n\left(\mathrm{H}_{2}\right) \sim 10^{5} \mathrm{~cm}^{-3}$. Considering an abundance ratio of $\left[\mathrm{H}_{2}\right] /\left[{ }^{13} \mathrm{CO}\right]=77 \times 10^{4}$ (Wilson \& Rood 1994), we estimate the $\mathrm{H}_{2}$ column density, $N\left(\mathrm{H}_{2}\right) \sim 2.1 \times 10^{23} \mathrm{~cm}^{-2}$. Finally, using the relation $M=\mu \mathrm{m}_{\mathrm{H}} d^{2} \Omega N\left(\mathrm{H}_{2}\right)$, where $\mu$ is the mean molecular weight per $\mathrm{H}_{2}$ molecule $(\mu \sim 2.72), m_{\mathrm{H}}$ the hydrogen atomic mass, $d$ the distance, and $\Omega$ the solid angle subtended by the structure, we calculate the total mass of the clump in $M \sim 10^{4} M_{\odot}$.

As an independent estimate, we derive the beam-averaged gas column density, the mass, and the volume density of the clump based on the dust continuum emission. In particular, we use the integrated flux of the continuum emission at $1.1 \mathrm{~mm}$ as obtained from The Bolocam Galactic Plane Survey II Catalog (BGPS II; Rosolowsky et al. 2010). The $1.1 \mathrm{~mm}$ continuum emission mostly originates in optically thin dust (Hildebrand 1983). Following Beuther et al. (2002a) and Hildebrand (1983), we calculate the mass and the gas column density of the clump using

$$
\begin{array}{r}
M_{\text {gas }}=\frac{1.3 \times 10^{-3}}{J_{v}\left(T_{\text {dust }}\right)} \frac{a}{0.1 \mu \mathrm{m}} \frac{\rho}{3 \mathrm{~g} \mathrm{~cm}^{-3}} \frac{R}{100} \frac{F_{v}}{\mathrm{Jy}} \\
\left(\frac{d}{\mathrm{kpc}}\right)^{2}\left(\frac{v}{2.4 \mathrm{THz}}\right)^{-3-\beta}\left[M_{\odot}\right]
\end{array}
$$

and

$$
\begin{array}{r}
N_{\text {gas }}=\frac{7.8 \times 10^{10}}{J_{v}\left(T_{\text {dust }}\right) \Omega_{\mathrm{b}}} \frac{a}{0.1 \mu \mathrm{m}} \frac{\rho}{3 \mathrm{~g} \mathrm{~cm}^{-3}} \frac{R}{100} \frac{F_{v}}{\mathrm{Jy}} \\
\left(\frac{v}{2.4 \mathrm{THz}}\right)^{-3-\beta}\left[\mathrm{cm}^{-2}\right]
\end{array}
$$

where $J_{v}\left(T_{\text {dust }}\right)=\left[\exp \left(h v / k T_{\text {dust }}\right)-1\right]^{-1}$ and $\Omega_{\mathrm{b}}, a, \rho, R$, and $\beta$ are the beam solid angle, grain size, grain mass density, gasto-dust ratio, and grain emissivity index for which we used the values of $\left(33^{\prime \prime}\right)^{2}$ in radians, $0.1 \mu \mathrm{m}, 3 \mathrm{~g} \mathrm{~cm}^{-3}, 100$, and 2 , respectively (Hunter 1997, Hunter et al. 2000, and Molinari et al. 2000). Based on the work of Sridharan et al. (2002) who derived dust temperatures ranging between 30 and $60 \mathrm{~K}$ for a sample of several massive star-forming regions, we adopt $T_{\text {dust }}=45 \mathrm{~K}$. For a distance $d=8.3 \mathrm{kpc}$ and an integrated flux intensity $F_{v}=5.18 \mathrm{Jy}$ at $1 \mathrm{~mm}$ (Rosolowsky et al. 2010), we obtain $N_{\text {gas }} \sim 4 \times 10^{23} \mathrm{~cm}^{-2}, M_{\text {gas }} \sim 8520 M_{\odot}$, and a volume density, $n\left(\mathrm{H}_{2}\right) \sim 1.4 \times 10^{5} \mathrm{~cm}^{-3}$. These values are in good agreement with those derived from the ${ }^{13} \mathrm{CO} J=3-2$ line using RADEX.

\subsection{Molecular outflows associated with G45.47}

As discussed in Sect. 3.1, the presence of spectral wings in the ${ }^{12} \mathrm{CO} J=3-2$ spectrum obtained towards G45.47 is a strong indicator that molecular outflow activity is taking place in the 

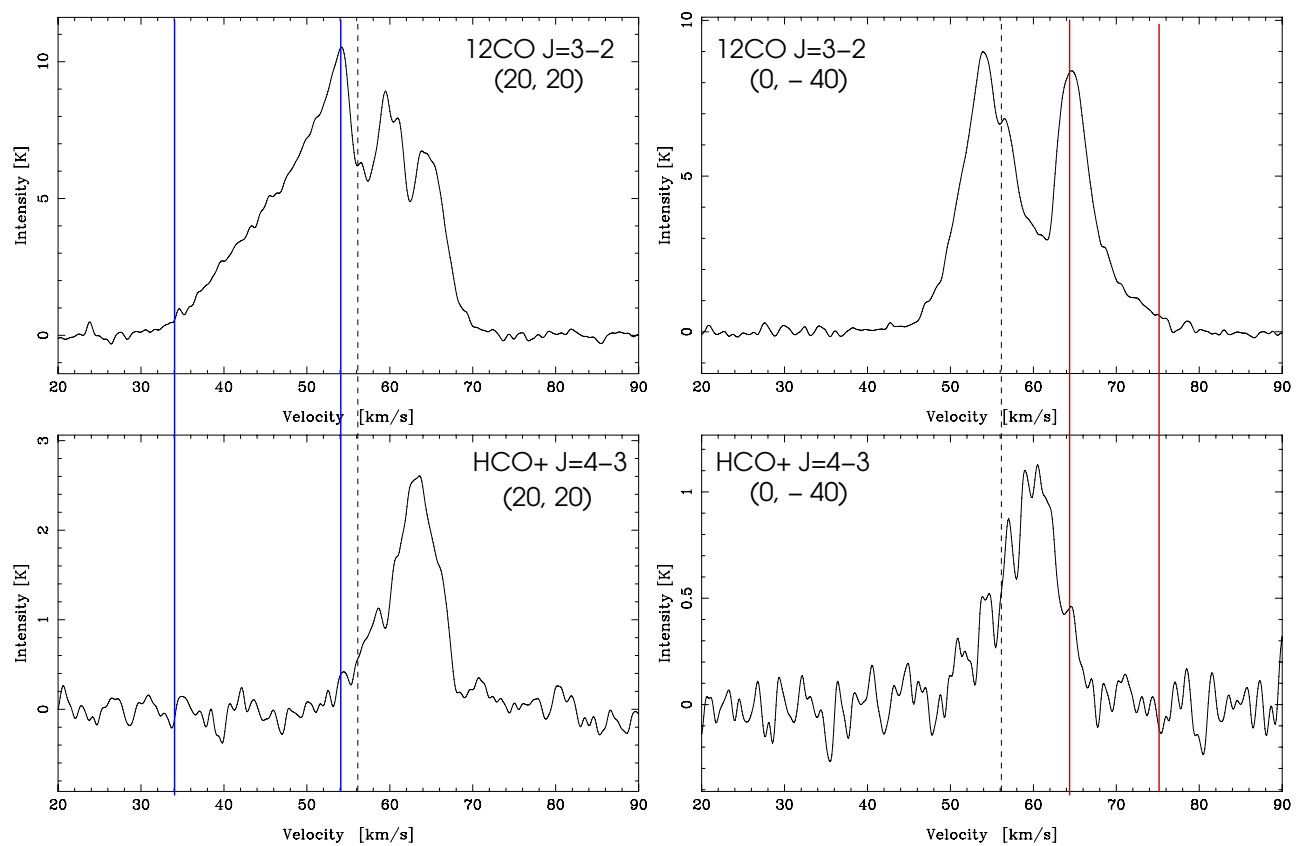

Fig. 10. Comparison between the ${ }^{12} \mathrm{CO} J=3-2$ and the $\mathrm{HCO}^{+} J=4-3$ spectra on the positions $(20,20)$ and $(0,-40)$. The dashed line indicates the systemic velocity. The velocity intervals corresponding to the blue and red wings are shown. The four spectra were Hanning smoothed.

region. To characterize the associated molecular outflows, it is first necessary to separate the outflowing gas from the molecular material of the clump, thereby identifying the velocity ranges related to each structure.

We consider two independent methods to determine these velocity ranges. The first method consists in identifying of the blue and red spectral wings based on a comparison between the ${ }^{12} \mathrm{CO} J=3-2$ and $\mathrm{HCO}^{+} J=4-3$ spectra. Figure 10 shows the comparison between both spectra on positions $(20,20)$ and $(0,-40)$ where the blue and red wings are largest. Considering emission up to about $2 \sigma$ of rms noise level, a blue and a red wing can be noticed in the ${ }^{12} \mathrm{CO}$ spectra between $\sim 34$ and $\sim 54$ and between $\sim 64$ and $\sim 75 \mathrm{~km} \mathrm{~s}^{-1}$, respectively.

The second method for identifying the molecular outflows is based on inspecting of the ${ }^{12} \mathrm{CO} J=3-2$ data cube, channel by channel, trying to spatially separate both outflows lobes. In Sect. 3.1 we mentioned two molecular features in the ${ }^{12} \mathrm{CO} J=3-2$ emission centered at RA $=19^{\mathrm{h}} 14^{\mathrm{m}} 27^{\mathrm{s}}$, Dec $=+11^{\circ} 09^{\prime} 45^{\prime \prime}(\mathrm{J} 2000)$ between $\sim 34$ and $\sim 54 \mathrm{~km} \mathrm{~s}^{-1}$ and at $\mathrm{RA}=19^{\mathrm{h}} 14^{\mathrm{m}} 26^{\mathrm{s}}$, Dec $=+11^{\circ} 08^{\prime} 45^{\prime \prime}(\mathrm{J} 2000)$ between $\sim 64$ and $\sim 75 \mathrm{~km} \mathrm{~s}^{-1}$, which we identify as the outflow's lobes related to G45.47 (see Fig. 9). These molecular features can be identified as the spectral wings detected in the ${ }^{12} \mathrm{CO} J=3-2$ spectra through the first method. Figure 11 shows a SpitzerIRAC three-color image $(3.6 \mu \mathrm{m}=$ blue, $4.5 \mu \mathrm{m}=$ green, and $8 \mu \mathrm{m}=$ red) of G45.47. The blue and red contours represent the ${ }^{12} \mathrm{CO} J=3-2$ emission integrated from $\sim 34$ to $54 \mathrm{~km} \mathrm{~s}^{-1}$ (blue lobe) and from $\sim 64$ to $75 \mathrm{~km} \mathrm{~s}^{-1}$ (red lobe).

Following Scoville et al. (1986), we estimate the ${ }^{12} \mathrm{CO}$ optical depth, $\tau_{12}$, of the gas in the molecular outflows using

$\frac{{ }^{12} T_{\mathrm{mb}}}{{ }^{13} T_{\mathrm{mb}}}=\frac{1-\exp \left(-\tau_{12}\right)}{1-\exp \left(-\tau_{12} / X\right)}$

where $X=\left[{ }^{12} \mathrm{CO}\right] /\left[{ }^{13} \mathrm{CO}\right]$ is the isotope abundance ratio. Using the relation $X=6.21 \times D_{\mathrm{GC}}+18.71$ (Milam et al. 2005), where $D_{\mathrm{GC}}=6.1 \mathrm{kpc}$ is the distance between G45.47 and the Galactic center, we obtain an isotope abundance ratio $X=57$ for this

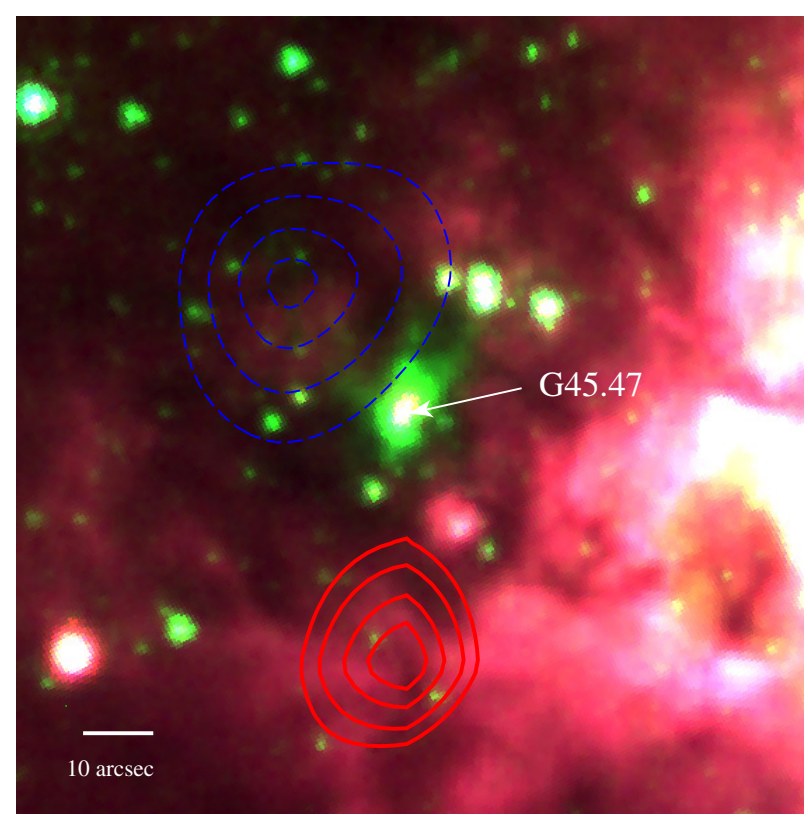

Fig. 11. Spitzer-IRAC three-color image $(3.6 \mu \mathrm{m}=$ blue, $4.5 \mu \mathrm{m}=$ green, and $8 \mu \mathrm{m}=$ red) of G45.47. The blue and red contours represent the ${ }^{12} \mathrm{CO} J=3-2$ emission integrated from 34 to $54 \mathrm{~km} \mathrm{~s}^{-1}$ (blue lobe), and from 64 to $75 \mathrm{~km} \mathrm{~s}^{-1}$ (red lobe). The blue contours are at 400 , 500,600 , and $670 \mathrm{~K} \mathrm{~km} \mathrm{~s}^{-1}$ and the red ones are at 140, 160, 180, and $200 \mathrm{~K} \mathrm{~km} \mathrm{~s}^{-1}$.

region. We adopt a constant $X$ throughout the outflows (Cabrit $\&$ Bertout 1990). Since we could not identify any blue wing in the ${ }^{13} \mathrm{CO}$ spectrum on position $(20,20)$, the ${ }^{12} T_{\mathrm{mb}} /{ }^{13} T_{\mathrm{mb}}$ ratio for both wings was estimated considering only the red one. We obtain a ${ }^{12} T_{\mathrm{mb}} /{ }^{13} T_{\mathrm{mb}}$ ratio of about 4.2 and derive a ${ }^{12} \mathrm{CO}$ optical depth of $\tau_{12} \sim 15$, which is adopted for both spectral wings in further calculations. 
We can now estimate the ${ }^{12} \mathrm{CO}$ column density of both outflow lobes from (see e.g. Buckle et al. 2010):

$N\left({ }^{12} \mathrm{CO}\right)=7.96 \times 10^{13} \mathrm{e}^{\frac{16.6}{T_{\mathrm{ex}}}} \frac{T_{\mathrm{ex}}+0.92}{1-\exp \left(\frac{-16.6}{T_{\mathrm{ex}}}\right) .} \int \tau_{12} \mathrm{~d} v$.

Taking into account that the ${ }^{12} \mathrm{CO} J=3-2$ is an optically thick transition $(\tau \geq 1)$, the integral can be approximated by

$\int \tau_{12} \mathrm{~d} v=\frac{1}{J\left(T_{\mathrm{ex}}\right)-J\left(T_{\mathrm{BG}}\right)} \frac{\tau_{12}}{1-\mathrm{e}^{-\tau_{12}}} \int{ }^{12} \mathrm{~T}_{\mathrm{mb}} \mathrm{d} v$

By integrating the ${ }^{12} \mathrm{CO}$ emission from 34 to $54 \mathrm{~km} \mathrm{~s}^{-1}$ and from 64 to $75 \mathrm{~km} \mathrm{~s}^{-1}$, we obtain $N(\mathrm{CO})_{\text {blue }} \sim 5.2 \times 10^{17} \mathrm{~cm}^{-2}$ and $N(\mathrm{CO})_{\text {red }} \sim 2 \times 10^{17} \mathrm{~cm}^{-2}$, respectively. The mass of each wing can be derived from the relation $M=\mu \mathrm{m}_{\mathrm{H}} d^{2} \Omega X(\mathrm{CO})^{-1} N(\mathrm{CO})$, where $X(\mathrm{CO})$ is the ${ }^{12} \mathrm{CO}$ relative abundance to $\mathrm{H}_{2}(X(\mathrm{CO}) \sim$ $7.4 \times 10^{-5}$ ). We obtain $\mathrm{M}_{\text {blue }} \sim 300 M_{\odot}$ and $M_{\text {red }} \sim 120 M_{\odot}$, yielding a total outflow mass of about $420 M_{\odot}$. In this way, the estimated total outflows mass represents about $4 \%$ of the clump mass, which agrees with the results of Beuther et al. (2002b), who established that approximately $4 \%$ of the clump gas is entrained in the molecular outflows.

The momentum and the kinetic energy of the wings can be derived from $P=M V_{\mathrm{c}}$ and $E_{\mathrm{k}}=0.5 M V_{\mathrm{c}}^{2}$, where $V_{\mathrm{c}}$ is a characteristic velocity estimated as the difference between the maximum velocity of detectable ${ }^{12} \mathrm{CO}$ emission in the wing and the systemic velocity of the gas $\left(\sim 56 \mathrm{~km} \mathrm{~s}^{-1}\right)$. Considering a $V_{\mathrm{c}}^{\text {blue }} \sim 22 \mathrm{~km} \mathrm{~s}^{-1}$ and a $V_{\mathrm{c}}^{\text {red }} \sim 19 \mathrm{~km} \mathrm{~s}^{-1}$, we obtain $P_{\text {blue }}=6.6 \times 10^{3} M_{\odot} \mathrm{km} \mathrm{s}^{-1}, P_{\text {red }}=2.3 \times 10^{3} M_{\odot} \mathrm{km} \mathrm{s}^{-1}$, $E_{\mathrm{k}}^{\text {blue }}=1.4 \times 10^{48} \mathrm{erg}$, and $E_{\mathrm{k}}^{\text {red }}=4.2 \times 10^{47} \mathrm{erg}$. Comparing our results with the work of Wu et al. (2004), who based on compiled data, concluded that the mass, momentum, and energy of molecular outflows range from $10^{-3}$ to $10^{3} M_{\odot}, 10^{-3}$ to $10^{4} M_{\odot} \mathrm{km} \mathrm{s}^{-1}$, and $10^{38}$ to $10^{48} \mathrm{erg}$, respectively, we can infer that in this work we are dealing with massive and energetic outflows.

Following Curtis et al. (2010), we define the dynamical time for the blue and red wings, $t_{\mathrm{dyn}}$, as the time for the bow shock travelling at the maximum velocity in the flow, $V_{\mathrm{c}}$, to travel the projected lobe length, $R_{\text {lobe }}$ :

$t_{\text {dyn }}=\frac{R_{\text {lobe }}}{V_{\mathrm{c}}}$

with $R_{\text {lobe }}^{\text {blue }}=1.8 \mathrm{pc}$ and $R_{\text {lobe }}^{\text {red }}=1.6 \mathrm{pc}$. These values were obtained by inspecting the blue and red lobe sizes in Fig. 11 and considering a distance of $8.3 \mathrm{kpc}$. We obtain a similar dynamical time of both lobes, $t_{\mathrm{dyn}}=0.8 \times 10^{5} \mathrm{yr}$. According to Beuther et al. (2002b), flow ages are good estimates of protostar lifetimes. We can then assume that the protostar responsible for the outflow activity has an age on the order of $10^{5} \mathrm{yr}$. This is consistent with the detection of ionized gas in the region, since to be able to ionize its surroundings the age of the protostar should at least be $10^{5} \mathrm{yr}$ (Sridharan et al. 2002).

\section{4. $\mathrm{HCO}^{+}$as tracer of molecular outflows}

As an independent test of out findings, we studied the $\mathrm{HCO}^{+}$ abundance towards $\mathrm{G} 45.47$. $\mathrm{HCO}^{+}$is believed to be the dominant ionized species in dense dark clouds (Rawlings et al. 2000; Dalgarno \& Lepp 1984), because it is very important for ionneutral chemistry. The ionization fraction of molecular clouds is a relevant parameter for studying the cloud chemistry and dynamics, hence the star-forming processes. Star formation occurs in the interior of dense cores, regions of high extinction where self-shielding prevents the UV photoionization of $\mathrm{H}_{2}$. Thus, cosmic ray ionization is believed to dominate photoionization in dense cores (McKee 1989). However, when YSOs are formed within the cores, the shocks and outflows produce deep changes in the chemistry, and photoionization processes are very likely. Indeed, in star-forming regions molecular enrichment occurs due to the desorption of molecular-rich ice mantles, followed by photochemical processing by shock-generated radiation fields. In particular, towards YSOs that are driving outflows, an enhancement in the $\mathrm{HCO}^{+}$abundance can be expected (Rawlings et al. $2000,2004)$. In what follows, we use a simple chemical network to estimate the $\mathrm{HCO}^{+}$abundance that would be produced by an standard cosmic ray ionization rate in the G45 clump in order to compare it with the abundance obtained from our observations.

The starting chemical reaction to form $\mathrm{HCO}^{+}$is the production of $\mathrm{H}_{3}^{+}$, mainly formed in the interaction between the cosmic rays (c.r.) and the molecular gas (e.g. Oka 2006):

$\mathrm{H}_{2}+$ c.r. $\rightarrow \mathrm{H}_{2}^{+}+\mathrm{e}^{-}$
$\mathrm{H}_{2}^{+}+\mathrm{H}_{2} \rightarrow \mathrm{H}_{3}^{+}+\mathrm{H}$.

The rate equation of this reaction is

$\zeta_{\mathrm{H}_{2}} n\left(\mathrm{H}_{2}\right)=\frac{\mathrm{d} n\left(\mathrm{H}_{3}^{+}\right)}{\mathrm{d} t}$,

where $\zeta_{\mathrm{H}_{2}}$ is the cosmic ray ionization rate and $n$ the density of the molecular species. By considering that the $\mathrm{HCO}^{+}$is mainly formed by the reaction of $\mathrm{H}_{3}^{+}$with $\mathrm{CO}$,

$\mathrm{H}_{3}^{+}+\mathrm{CO} \rightarrow \mathrm{HCO}^{+}+\mathrm{H}_{2}$,

and destroyed through recombination with electrons,

$\mathrm{HCO}^{+}+\mathrm{e}^{-} \rightarrow \mathrm{CO}+\mathrm{H}$

the rate equations can be equated, leading to

$k_{\mathrm{HCO}^{+}} n\left(\mathrm{H}_{3}^{+}\right) n(\mathrm{CO})=k_{\mathrm{CO}} n\left(\mathrm{HCO}^{+}\right) n(\mathrm{e})$,

where $k_{\mathrm{HCO}^{+}}$and $k_{\mathrm{CO}}$ are the coefficient rates and $n(\mathrm{e})$ the electron density. Assuming that the main destruction mechanism for $\mathrm{H}_{3}^{+}$is through the formation of $\mathrm{HCO}^{+}$, the rate of destruction of $\mathrm{H}_{3}^{+}$is therefore equal to the formation rate of $\mathrm{HCO}^{+}$. This implies that the left-hand side of Eq. (10) is equal to right-hand side of Eq. (13). Then, it is possible to write an expression for the cosmic ionization rate as a function of the molecular densities:

$\zeta_{\mathrm{H}_{2}}=k_{\mathrm{CO}} \frac{n\left(\mathrm{HCO}^{+}\right) n(\mathrm{e})}{n\left(\mathrm{H}_{2}\right)}$.

The rate coefficient $k_{\mathrm{CO}}$, extracted from the UMIST database (Woodall et al. 2007), is $k_{\mathrm{CO}}=2.4 \times 10^{-7}(T / 300 \mathrm{~K})^{-0.69}$. Equation (14) can be approximated using the column densities, leading to

$\zeta_{\mathrm{H}_{2}} \simeq k_{\mathrm{CO}} \frac{N\left(\mathrm{HCO}^{+}\right) X(\mathrm{e}) n\left(\mathrm{H}_{2}\right)}{N\left(\mathrm{H}_{2}\right)}$,

where $X(\mathrm{e})$ is the electron abundance. Using this equation and assuming typical values for the cosmic ionization rate and electron abundance of $\zeta_{\mathrm{H}_{2}}=(1-5) \times 10^{-17} \mathrm{~s}^{-1}$ (Dalgarno 2006) and $X(\mathrm{e}) \sim 10^{-7}$ (Bergin et al. 1999), respectively, we derive the $\mathrm{HCO}^{+}$column density. We use $N\left(\mathrm{H}_{2}\right)=2 \times 10^{23} \mathrm{~cm}^{-2}$ and $n\left(\mathrm{H}_{2}\right)=1 \times 10^{5} \mathrm{~cm}^{-3}$, as derived above, and we assume $T=20 \mathrm{~K}$. Finally, we find that the $N\left(\mathrm{HCO}^{+}\right)$should be in the 
range $(1.5-6.5) \times 10^{14} \mathrm{~cm}^{-2}$, leading to an abundance $X\left(\mathrm{HCO}^{+}\right)$ in between $6.5 \times 10^{-10}$ and $3.2 \times 10^{-9}$.

On the other hand, we analyze the central $\mathrm{HCO}^{+} J=4-3$ spectrum and use the RADEX code (van der Tak et al. 2007) to derive its column density. Assuming background and kinetic temperatures of $2.73 \mathrm{~K}$ and $20 \mathrm{~K}$, respectively, and the same $\mathrm{H}_{2}$ density as used above, we varied the $\mathrm{HCO}^{+}$column density until obtaining a good fit for the observed peak temperature. The best fit was obtained with $N\left(\mathrm{HCO}^{+}\right) \sim 1.5 \times 10^{15} \mathrm{~cm}^{-2}$, leading to an abundance of $X\left(\mathrm{HCO}^{+}\right) \sim 7.5 \times 10^{-9}$. This value, more than twice greater than the higher value obtained above, might indicate that the cosmic ray ionization is insufficient to produce the observed $\mathrm{HCO}^{+}$abundance through the described chemical network. Therefore, following Rawlings et al. (2000, 2004), we suggest that the observed $\mathrm{HCO}^{+}$abundance must be mostly produced by the outflowing activity in the region. In spite of the uncertainties in the calculations, this is an independent proof to support a scenario with outflows in the region.

\subsection{Looking for the driving source of the molecular outflows}

Wilner et al. (1996) report $5^{\prime \prime}$ resolution observations of the $\mathrm{HCO}^{+} J=1-0$ transition towards $\mathrm{G} 45.47$ obtained with the OVRO millimeter array. They identify at least five $\mathrm{HCO}^{+} J=$ 1-0 clumps and suggest that the formation of an OB cluster would be taking place in the region. However, the authors did not carry out any search of infrared point sources in the region to confirm that hypothesis. In this context, we wonder if the massive molecular outflows observed towards G45.47 were caused by one or more stars. Based on a near-infrared analysis, we searched for the driving source candidates of the massive molecular outflows.

Figure 12 shows the radio continuum emission at $6 \mathrm{~cm}$ obtained from The Multi-Array Galactic Plane Imaging Survey (MAGPIS; White et al. 2005) of the area mapped using ASTE. The green contours represent the $\mathrm{HCO}^{+} J=4-3$ emission distribution integrated between 58 and $67 \mathrm{~km} \mathrm{~s}^{-1}$. The black contours were taken from the paper of Wilner et al. (1996) and represent the $\mathrm{HCO}^{+} J=1-0$ emission distribution integrated in the same velocity interval. The region observed by Wilner et al. (1996) is indicated. The five clumps reported by the authors have been labeled. The positional coincidence among the center of the $\mathrm{HCO}^{+} J=4-3$ clump, the clump 3 of $\mathrm{HCO}^{+} J=1-0$, and a radio continuum source, which has been identified as the UCHII region G45.47, is striking. The location of the YSO candidates in the observed region is indicated with red circles that correspond to the red circles in Fig. 13. Among the five YSO candidates projected onto the $\mathrm{HCO}^{+} J=4-3$ emission, 2MASS $19142564+1109283$ is the only one having a positional coincidence with a clump of $\mathrm{HCO}^{+} J=1-0$ (clump 3 ). We do not find any embedded infrared sources in the other four clumps, suggesting that these clumps could be in a prestellar core stage or that they might be tracing the origin of the molecular outflows. We suggest that the most likely candidate to be the driving source of the molecular outflows is 2MASS 19142564+ 1109283.

Figure 13 shows a color-color diagram (CCD) including all the 2MASS point sources located in the observed region. The sources with infrared excess (YSO candidates) and the main sequence or giant star candidates are indicated with red and blue circles, respectively. The reddenest object in the region is 2MASS 19142564+1109283.

To characterize the infrared source 2MASS 19142564+ 1109283 , we perform a fitting of its spectral energy distribution

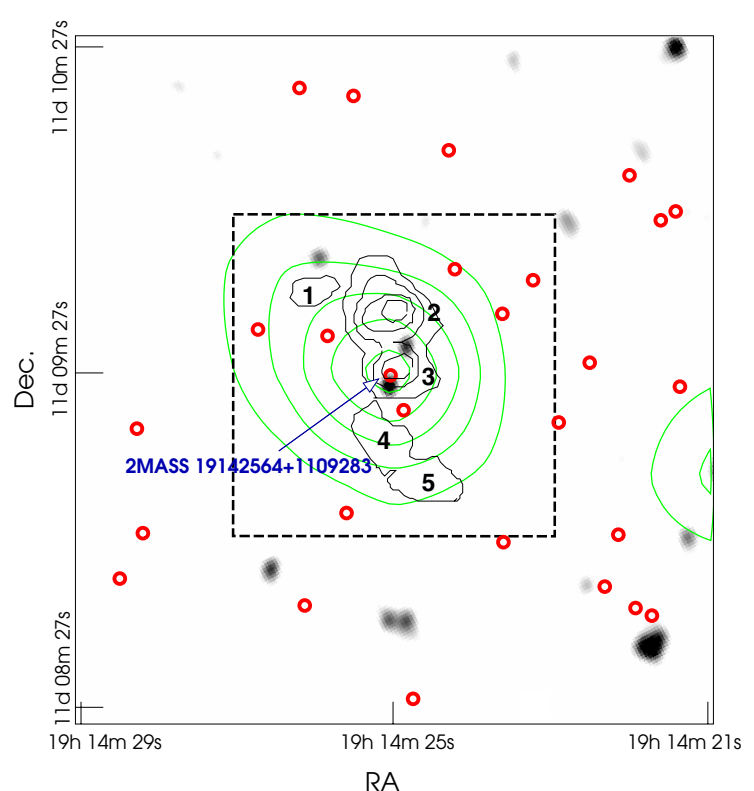

Fig. 12. Radio continuum emission at $6 \mathrm{~cm}$ (MAGPIS; White et al. 2005) of the area mapped using ASTE. The green contours represent the $\mathrm{HCO}^{+} J=4-3$ emission distribution integrated between 58 and $67 \mathrm{~km} \mathrm{~s}^{-1}$. The black contours were taken from the paper of Wilner et al. (1996) and represent the $\mathrm{HCO}^{+} J=1-0$ emission distribution integrated from 58 and $67 \mathrm{~km} \mathrm{~s}^{-1}$. The box indicates the area observed by Wilner et al. (1996). The numbers show the five $\mathrm{HCO}^{+} J=1-0$ clumps. The red circles represent the YSO candidates shown in Fig. 13.

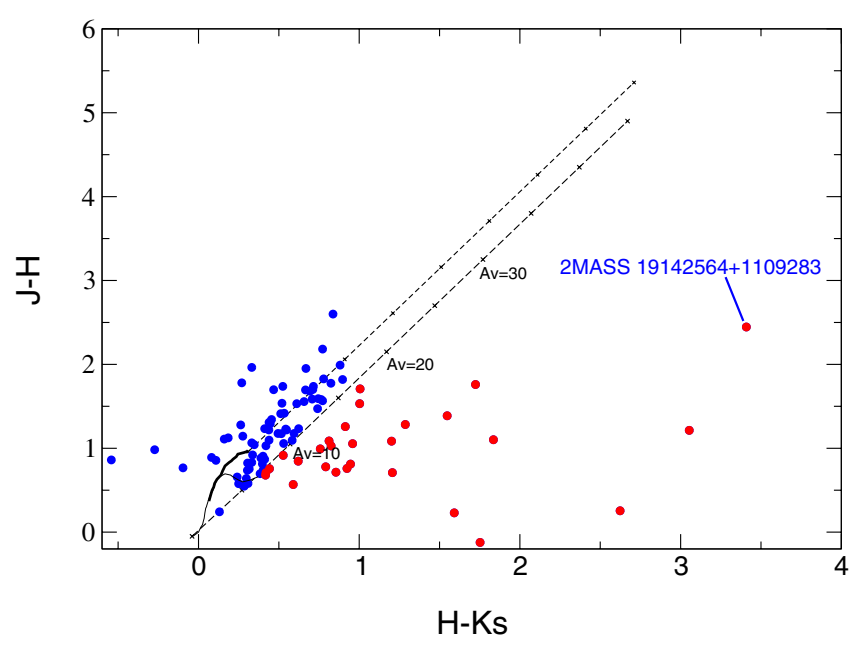

Fig. 13. Color-color diagram of the 2MASS infrared sources in the vicinity of G45.47. The two solid curves represent the location of the main sequence (thin line) and the giant stars (thick line) derived from Bessell \& Brett (1988). The parallel dashed lines are reddening vectors with the crosses placed at intervals corresponding to five magnitudes of visual extinction. We have assumed the interstellar reddening law of Rieke \& Lebofsky (1985) $\left(A_{J} / A_{V}=0.282 ; A_{H} / A_{V}=0.175\right.$ and $\left.A_{K} / A_{V}=0.112\right)$. The sources reddened by circumstellar dust are indicated as red circles.

(SED) using the tool developed by Robitaille et al. (2007) ${ }^{5}$. We adopt an interstellar extinction in the line of sight, $A_{\mathrm{v}}$, between 5 and $17 \mathrm{mag}$. The range of $A_{\mathrm{v}}$ is chosen by inspecting the location in an $[H-K]$ vs. $[J-H]$ CCD of the 2 MASS sources in the region (see Fig. 13). To construct the SED we consider the fluxes at the JHK 2MASS bands, Spitzer-IRAC 5.8 and $8 \mu \mathrm{m}$ bands,

http://caravan.astro.wisc.edu/protostars/ 


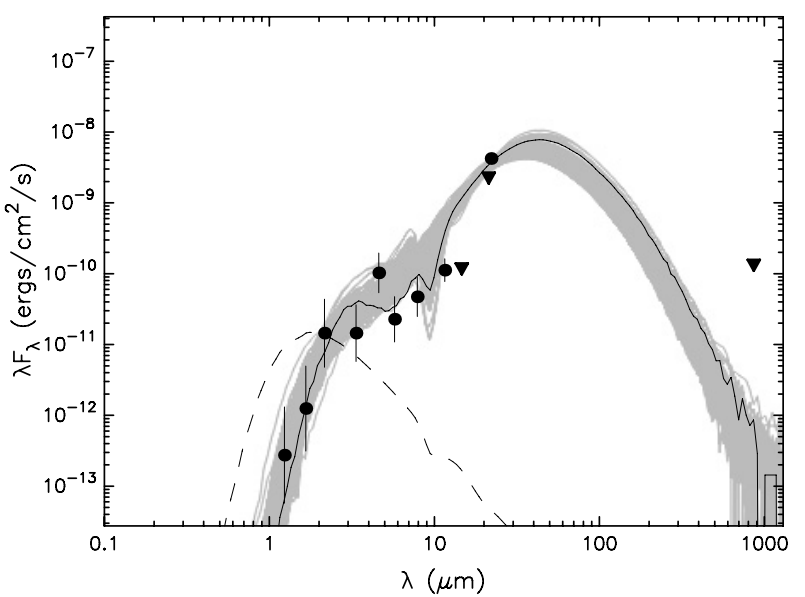

Fig. 14. Best-fitting SED models for G45.47. The filled circles indicate the measured fluxes at the JHK 2MASS bands, Spitzer-IRAC bands at 5.8 and $8.0 \mu \mathrm{m}$, and the WISE bands at 3.4, 4.6, 12, and $22 \mu \mathrm{m}$. Triangles indicate fluxes considered as upper limits, as MSX bands at 14 and $21 \mu \mathrm{m}$ and the SCUBA band at $850 \mu \mathrm{m}$. Black and gray solid curves represent the best-fit model and the subsequent good fittings, respectively. The dashed line shows the best-fit model for a central source contribution in absence of circumstellar dust.

WISE 3.4, 4.6, 12, and $22 \mu \mathrm{m}$ bands, MSX 14 and $21 \mu \mathrm{m}$ bands, and SCUBA $850 \mu \mathrm{m}$ band. The fluxes of those datasets having lower angular resolution (MSX and SCUBA) were considered as upper limits. Figure 14 shows the best-fitting SEDs models for 2MASS 19142564+1109283. We select models that satisfies the condition

$\chi^{2}-\chi_{\text {best }}^{2}<2 N$

where $\chi_{\text {best }}^{2}$ is the minimum value of the $\chi^{2}$ among all models, and $N$ the number of input data fluxes (fluxes specified as upper limit do not contribute to $N$ ). Hereafter, we refer to models satisfying Eq. (16) as "selected models".

From the SED fitting, several model parameters can be obtained and, as expected, some can be better constrained than others. Taking the values yielded by the "selected models" for several parameters into account, we derive the following main results:

- the total luminosity distribution of the source has an average value of $2 \times 10^{4} L_{\odot}$ with a spread between $8 \times 10^{3}$ and $5 \times$ $10^{4} L_{\odot}$;

- embedded in the molecular clump there is a massive protostar of about $15 M_{\odot}$ (early B-type star) accreting material from its envelope at high rates, $\dot{M}_{\text {env }} \sim 2 \times 10^{-4} M_{\odot} \mathrm{yr}^{-1}$;

- despite the large spread in the central source's age distribution that goes from $2 \times 10^{3}$ to $10^{6} \mathrm{yr}$, it peaks at about $3 \times 10^{5} \mathrm{yr}$, as expected for protostars that have begun to ionize their surroundings. Besides this, this result agrees with the dynamical time, $t_{\mathrm{dyn}}=1 \times 10^{5} \mathrm{yr}$, derived in Sect. 3.3.

\section{Summary}

We carried out a study of the UCHII region G045.47+0.05 and its surroundings based on molecular line observations and public infrared data. We find a molecular gas condensation associated with G045.47+0.05. The detection of the CS $J=7-6$ transition reveals that the UCHII region is still embedded in warm and dense molecular gas. The CS spectrum obtained towards the position $(0,0)$ has a pronounced self-absorption dip at about
$59 \mathrm{~km} \mathrm{~s}^{-1}$ with the red velocity component more intense than the blue one. This asymmetry in the CS profile suggests an expansion of the molecular gas.

All molecular transitions have the same main three velocity components with a self-absorption dip at about $58-59 \mathrm{~km} \mathrm{~s}^{-1}$ that correspond to the central velocity of the molecular cloud GRSMC G045.49+00.04 where G45.47 is embedded.

Based on the ratio between the ${ }^{12} \mathrm{CO}$ and ${ }^{13} \mathrm{CO} J=3-2$ transitions, we estimated the ${ }^{13} \mathrm{CO}$ opacity, $\tau_{13}$, towards the center of the clump is about 1.9 , revealing that this line is optically thick. This result agrees with the structure exhibited by the ${ }^{13} \mathrm{CO}$ spectrum towards the center of G045.47+0.05.

Using the RADEX code we derive a ${ }^{13} \mathrm{CO} J=3-2$ column density and $\mathrm{H}_{2}$ volume density of about $2.8 \times 10^{17} \mathrm{~cm}^{-2}$ and $10^{5} \mathrm{~cm}^{-3}$, respectively. The $\mathrm{H}_{2}$ column density and the total mass of the clump were estimated as $2.1 \times 10^{23} \mathrm{~cm}^{-2}$ and $M \sim 10^{4} M_{\odot}$, respectively. From an independent estimate based on the dust continuum emission at $1 \mathrm{~mm}$ we derive $N_{\text {gas }} \sim 4 \times 10^{23} \mathrm{~cm}^{-2}, M_{\text {gas }} \sim 8520 M_{\odot}$, and a volume density, $n\left(\mathrm{H}_{2}\right) \sim 1.4 \times 10^{5} \mathrm{~cm}^{-3}$, in good agreement with the values derived from the molecular lines.

From the analysis of the ${ }^{12} \mathrm{CO} J=3-2$ and $\mathrm{HCO}^{+}$transitions, we report molecular outflows related to the UCHII region G045.47+0.05. We estimate the blue and red lobe masses as about 300 and $120 M_{\odot}$, respectively. The total mass of the outflows represents about $4 \%$ of the clump mass. The dynamical time of both lobes, a good indicator of the protostar age, was estimated as about $8 \times 10^{4} \mathrm{yr}$. This agrees with the presence of ionized gas around the central object.

Based on infrared data we searched for the protostar(s) that must be driving the outflows. We find that the source 2MASS $19142564+1109283$ is a YSO candidate located at the center of the clump, coinciding with the radio continuum emission associated with the UCHII region. Besides this, this infrared source is the only YSO candidate related to one of the five $\mathrm{HCO}^{+}$ clump detected by Wilner et al. (1996). We did not detect any YSO candidate associated with the four other $\mathrm{HCO}^{+}$clumps. This result suggests that the massive molecular outflows are generated by the protostar 2MASS $19142564+1109283$.

Finally, from a spectral energy distribution analysis of the source we derived a total luminosity, protostar mass, and protostar age of about $2 \times 10^{4} L_{\odot}, 15 M_{\odot}$ (early B-type star), and $3 \times 10^{5} \mathrm{yr}$, respectively.

Massive molecular outflows give, on large scales, a good piece of information about the physical processes taking place in the innermost parts of the star-forming cores. Their detection gives indirect evidence that massive star formation is a scaledup version of low-mass star formation. The identification of a massive protostar that would be generating the massive outflows reinforces this scenario.

Acknowledgements. We wish to thank the referee, Dr. Herpin, whose constructive criticism has helped make this a better paper. M.O., S.P., S.C., and G.D. are members of the Carrera del Investigador Científico of CONICET, Argentina. This work was partially supported by Argentina grants awarded by Universidad de Buenos Aires, CONICET, and ANPCYT. M.R. wishes to acknowledge support from FONDECYT (CHILE) grant No108033. She is supported by the Chilean Center for Astrophysics FONDAP No. 15010003. The ASTE project is driven by Nobeyama Radio Observatory (NRO), a branch of the National Astronomical Observatory of Japan (NAOJ), in collaboration with University of Chile, and Japanese institutes including the University of Tokyo, Nagoya University, Osaka Prefecture University, Ibaraki University, Hokkaido University, and Joetsu University of Education. 


\section{References}

Bergin, E. A., Plume, R., Williams, J. P., \& Myers, P. C. 1999, ApJ, 512, 724 Bessell, M. S., \& Brett, J. M. 1988, PASP, 100, 1134

Beuther, H. 2002, Ph.D. Thesis, Max-Planck-Institut für Radioastronomie Beuther, H., Schilke, P., Menten, K. M., et al. 2002a, ApJ, 566, 945

Beuther, H., Schilke, P., Sridharan, T. K., et al. 2002b, A\&A, 383, 892

Bonnell, I. A., \& Bate, M. R. 2006, MNRAS, 370, 488

Buckle, J. V., Curtis, E. I., Roberts, J. F., et al. 2010, MNRAS, 401, 204

Cabrit, S., \& Bertout, C. 1990, ApJ, 348, 530

Caswell, J. L., Vaile, R. A., Ellingsen, S. P., Whiteoak, J. B., \& Norris, R. P. 1995, MNRAS, 272, 96

Curtis, E. I., Richer, J. S., Swift, J. J., \& Williams, J. P. 2010, MNRAS, 408, 1516

Cyganowski, C. J., Whitney, B. A., Holden, E., et al. 2008, AJ, 136, 2391

Cyganowski, C. J., Brogan, C. L., Hunter, T. R., \& Churchwell, E. 2009, ApJ, 702,1615

Dalgarno, A. 2006, Proc. National Academy of Science, 1031, 12269

Dalgarno, A., \& Lepp, S. 1984, ApJ, 287, L47

Ezawa, H., Kawabe, R., Kohno, K., \& Yamamoto, S. 2004, in SPIE Conf. Ser. 5489, ed. J. M. Oschmann Jr., 763

Fazio, G. G., Hora, J. L., Allen, L. E., et al. 2004, ApJS, 154, 10

Giveon, U., Becker, R. H., Helfand, D. J., \& White, R. L. 2005a, AJ, 129, 348

Giveon, U., Becker, R. H., Helfand, D. J., \& White, R. L. 2005b, AJ, 130, 156

Hildebrand, R. H. 1983, QJRAS, 24, 267

Hiramatsu, M., Hayakawa, T., Tatematsu, K., et al. 2007, ApJ, 664, 964

Hofner, P., Peterson, S., \& Cesaroni, R. 1999, ApJ, 514, 899

Hunter, T. R. 1997, Ph.D. Thesis, Smithsonian Astrophysical Observatory, 60 Garden St. MS-78, Cambridge, MA 02178, USA

Hunter, T. R., Phillips, T. G., \& Menten, K. M. 1997, ApJ, 478, 283

Hunter, T. R., Churchwell, E., Watson, C., et al. 2000, AJ, 119, 2711

Kuchar, T. A., \& Bania, T. M. 1994, ApJ, 436, 117

Lehtinen, K. 1997, A\&A, 317, L5

Leung, C. M. 1978, ApJ, 225, 427

Matthews, H. E., Goss, W. M., Winnberg, A., \& Habing, H. J. 1977, A\&A, 61, 261
McKee, C. F. 1989, ApJ, 345, 782

Milam, S. N., Savage, C., Brewster, M. A., Ziurys, L. M., \& Wyckoff, S. 2005, ApJ, 634, 1126

Molinari, S., Brand, J., Cesaroni, R., \& Palla, F. 2000, A\&A, 355, 617

Oka, T. 2006, Proc. National Academy of Science, 1031, 12235

Paron, S., Ortega, M. E., Rubio, M., \& Dubner, G. 2009, A\&A, 498, 445

Peretto, N., \& Fuller, G. A. 2009, A\&A, 505, 405

Qin, S.-L., Wang, J.-J., Zhao, G., Miller, M., \& Zhao, J.-H. 2008, A\&A, 484, 361

Rathborne, J. M., Jackson, J. M., \& Simon, R. 2006, ApJ, 641, 389

Rathborne, J. M., Johnson, A. M., Jackson, J. M., Shah, R. Y., \& Simon, R. 2009, ApJS, 182, 131

Rawlings, J. M. C., Taylor, S. D., \& Williams, D. A. 2000, MNRAS, 313, 461

Rawlings, J. M. C., Redman, M. P., Keto, E., \& Williams, D. A. 2004, MNRAS, 351,1054

Rieke, G. H., \& Lebofsky, M. J. 1985, ApJ, 288, 618

Robitaille, T. P., Whitney, B. A., Indebetouw, R., \& Wood, K. 2007, ApJS, 169, 328

Rosolowsky, E., Dunham, M. K., Ginsburg, A., et al. 2010, ApJS, 188, 123

Scoville, N. Z., Sargent, A. I., Sanders, D. B., et al. 1986, ApJ, 303, 416

Sridharan, T. K., Beuther, H., Schilke, P., Menten, K. M., \& Wyrowski, F. 2002, ApJ, 566, 931

Tan, J. C., \& McKee, C. F. 2002, in Hot Star Workshop III: The Earliest Phases of Massive Star Birth, ed. P. Crowther, ASP Conf. Ser., 267, 267

van der Tak, F. F. S., Black, J. H., Schöier, F. L., Jansen, D. J., \& van Dishoeck, E. F. 2007, A\&A, 468, 627

White, R. L., Becker, R. H., \& Helfand, D. J. 2005, AJ, 130, 586

Wilner, D. J., Ho, P. T. P., \& Zhang, Q. 1996, ApJ, 462, 339

Wilson, T. L., \& Rood, R. 1994, ARA\&A, 32, 191

Wood, D. O. S., \& Churchwell, E. 1989, ApJS, 69, 831

Woodall, J., Agúndez, M., Markwick-Kemper, A. J., \& Millar, T. J. 2007, A\&A, 466, 1197

Wu, Y., Wei, Y., Zhao, M., et al. 2004, A\&A, 426, 503

Zhou, S. 1992, ApJ, 394, 204 